\title{
An Evaluation of the Applicability of Water Model Testing to Liquid Metal Engineering Problems
}

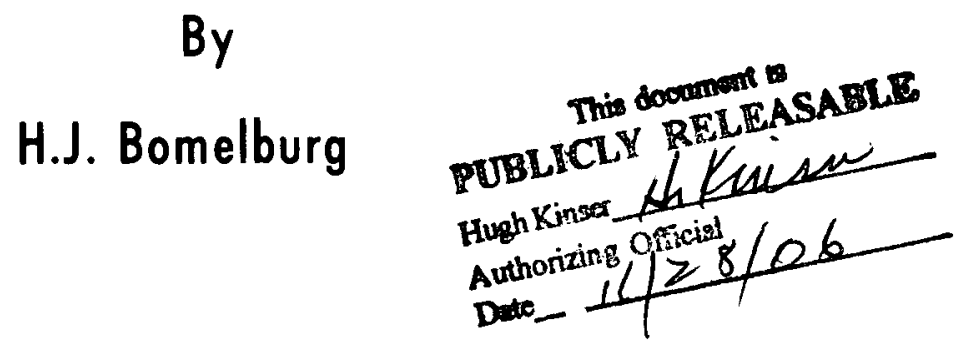

LEGAL NOTICE

This report was prepared as an account of Government sponsored work. Neither the United States, nor the Commission, nor any person acting on behalf of the Commission:

A. Makes any warranty or representation, expressed or implied, with respect to the accuracy, completeness, or usefulness of the information contained in this report, or that the use of any information, apparatus, method, or process disclosed in this report may not infringe privately owned rights; or

B. Assumes any liabilities with respect to the use of, or for damages resulting from the use of any information, apparatus, method, or process disclosed in this report.

As used in the above. "person acting on behalf of the Commission" includes any empioyee or contractor of the Commission, or employee of such contractor, to the extent that such employee or contractor of the Commission, or employee of such contractor prepares disseminates, or provides access to, any information pursuant to his employment or contract with the Commission, or his employment with such contractor.

\section{Liquid Metal Engineering Center \\ Operated for \\ the U.S. Atomic Energy Commission by Atomics International}




\section{DISCLAIMER}

This report was prepared as an account of work sponsored by an agency of the United States Government. Neither the United States Government nor any agency Thereof, nor any of their employees, makes any warranty, express or implied, or assumes any legal liability or responsibility for the accuracy, completeness, or usefulness of any information, apparatus, product, or process disclosed, or represents that its use would not infringe privately owned rights. Reference herein to any specific commercial product, process, or service by trade name, trademark, manufacturer, or otherwise does not necessarily constitute or imply its endorsement, recommendation, or favoring by the United States Government or any agency thereof. The views and opinions of authors expressed herein do not necessarily state or reflect those of the United States Government or any agency thereof. 


\section{DISCLAIMER}

Portions of this document may be illegible in electronic image products. Images are produced from the best available original document. 


\section{DISTRIBUTION}

This report has been distributed according to the category "General, Miscellaneous and Progress Reports" as given in "Standard Distribution Lists for Unclassified Scientific and Technical Reports," TID -4500 . 


\section{CONTENTS}

Page

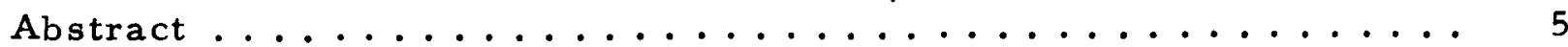

I. Introduction $\ldots \ldots \ldots \ldots \ldots \ldots \ldots \ldots \ldots \ldots \ldots \ldots \ldots \ldots \ldots \ldots$

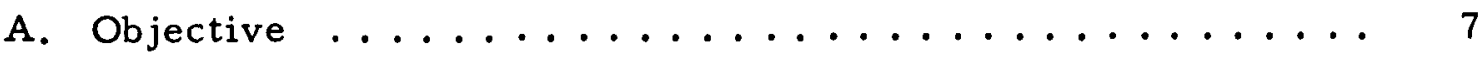

B. Approach ...................... 8

C. Summary...................... 8

I. Dimensional Analysis as Applied to Model Testing for

Liquid Metals .......................... 11

III. Property Differences Between Water and Liquid Metals . . . . 13

IV. Model Testing of Flow in Pipes, Reactor Cores and

Similar Geometries .................... 16

V. Model Testing of Pumps................... 27

VI. Model Testing of Bearings ................... 39

VII. Model Testing of Fluid Seals. . . . . . . . . . . . . 51

VIII. Liquid Metal Heat Transfer Problems and Model Testing . . . . 53

A. Forced Convection Heat Transfer. . . . . . . . . . . 53

B. Heat Transfer by Natural Convection ............. 60

C. Two-Phase Flow and Heat Transfer ............ 62

D. Summary on Heat Transfer . . . . . . . . . . . 65

IX. Special Flow Problems and Their Model Testing ........ 67

A. Cavitation ...................... 67

B. Vortexing ........................... 69

C. Valves and Flowmeters ............... 71

D. Flow Transients ..................... 73

X. Conclusions and Recommendations. . . . . . . . . . . 79

References .......................... 83

\section{FIGURES}

1. Dynamic Viscosity of Water and of Liquid Sodium . . . . . . . 14

2. Kinematic Viscosity of Water and Liquid Sodium .......... 15

3. Dynamic Pressure (Velocity Head) as a Function of Velocity ..... 18

4. Vapor Pressure of Water and of Liquid Sodium ........... 32 


\begin{abstract}
The extent to which water model testing can be substituted for liquid metal tests in investigating liquid metal flow problems is examined by applying dimensional analysis. The following problems are considered in detail: flow through piping systems, pumps, bearings, and fluid seals, as well as flow problems connected with liquid metal heat transfer, cavitation, vortexing, and transients.
\end{abstract}




\section{INTRODUCTION}

Liquid metals are to be used in the future as cooling fluids in large fast breeder reactors. The various components in the coolant circuits of such reactors must be efficient, highly reliable, and long lived. These requirements impose very strict demands on the quality of the design and fabrication of the components. Consequently, it is normal practice to thoroughly test a new design before it is accepted for actual service. However, testing in liquid metals can become quite involved, time consuming, and expensive; therefore, water is often considered as a substitute test fluid. As the properties of water differ from those of liquid metals, it is conceivable that water test results may not be directly applicable to liquid metals.

Over the last century, fluid mechanical testing techniques have been developed to a fairly high degree of sophistication, particularly with water and air. Models, substantially scaled down from the prototype, have most of ten been used for such purposes. It would therefore be of great value if these well established techniques could be applied effectively in the development of the rather unique field of liquid metal technology.

\section{A. OBJECTIVE}

The general objective of this study is to investigate the extent to which water model tests can be substituted for liquid metal tests in verifying the design of a particular component which is part of a liquid metal flow system. This is done from the theoretical viewpoint of fluid mechanics and also with regard to any previous practical experience in this field.

The various components which normally form the liquid metal cooling sys tem of a fast breeder reactor are investigated individually; in each case a determination is made as to the effectiveness of a water test. Principal nondimensional parameters are presented where applicable, as they frequently serve to facilitate the transfer of water test data to the actual liquid metal case. Sources of error and accuracy limits are defined where possible. Recommendations for performing useful water model tests are given in cases where the state-of-the-art is far enough advanced. For this purpose the latest publications in the field are reviewed. An extensive reference list is included as a guide for further study. 


\section{B. APPROACH}

The methods of dimensional analysis are described, and the way in which they are applied to derive dimensionless parameters which are important for model testing is shown. Because the properties of either water or liquid metals are factors in these parameters, these properties are compared on a quantitative basis.

Being the most straightforward application, the testing of flow through straight pipes is considered. This is then extended to more complicated flow patterns such as the flow through rough or curved ducts, through reactor cores, etc.

Flow through pumps is considered next. Here the various nondimensional pump parameters are derived and evaluated as to their practical usefulness and importance for testing purposes. Cavitation testing of pumps is also investigated.

Testing of pump bearings (liquid-metal-lubricated journal bearings) is investigated; liquid metal bearing technology apparently is still in its infancy and testing of bearings might therefore become quite important in the near future.

Since the solution to heat transfer problems is very important for fast breeder reactors, the possibility of using water in heat transfer testing procedures is investigated, although it must be borne in mind from the beginning that water certainly does not resemble liquid metals at all as far as heat trans fer is concerned.

Finally, a number of special flow problems (most of which are highly complex and for which no satisfactory theory exists as yet) are investigated in spite of the fact that they are also not readily accessible to model testing.

\section{SUMMARY}

Fluid flow phenomena in liquid metal systems can be investigated with various degrees of success by water model testing. Nondimensional parameters are extremely helpful in reducing the amount of testing, in presenting the test data in an expedient way, and in directly predicting corresponding data for moderately different conditions (e.g., if water were substituted for liquid metals or vice versa). 
Whereas the Reynolds number (Re-number) is often the most important similarity parameter for many flow conditions (particularly for flow in ducts), it is not enough merely to simulate Re-numbers if liquid metal flow is to be investigated by a corresponding water flow. Certain flow conditions (such as the flow through pumps, bearings, etc.) require the simulation of other additional similarity parameters. Hence, it is not always feasible to simulate all pertinent similarity parameters simultaneously. The parameter whose influence is clearly the most important is usually simulated, therefore. In other cases (as in heat transfer) the requirements with respect to simulation of pertinent dimensionless parameters are sometimes so conflicting that it is impractical to substitute water for liquid metals in model testing.

In an indirect approach, however, it is nevertheless sometimes possible to use water tests to check on the validity of a new theoretical technique. If such a new theory can be verified by water tests, the confidence level for its successful applicability to liquid metals is usually increased considerably.

If, finally, the flow situation is so complicated (as in two-phase flows, etc.) that a good theoretical understanding is not available, it might still be feasible to use water testing if valid correlations between water test data and liquid metal test data have been successfully established by a series of corresponding tests, both in water and in liquid metal. This method must be considered as a last resort because it usually involves a great deal of expensive test work.

Unfortunately, accurate model testing is generally possible only for flow conditions that are well understood theoretically, which implies that conditions that pose theoretical problems are usually also less accessible to model testing. 


\section{DIMENSIONAL ANALYSIS AS APPLIED TO MODEL TESTING FOR LIQUID METALS}

In order to test the hydrodynamic behavior of liquid metals in various components (such as pumps, fuel assemblies, etc.) such tests are often first conducted in water. Water model tests are normally much easier to conduct and are therefore less time consuming and less expensive. The technical justification for this substitution is usually based on reasons of dimensional analysis or similitude." However, it is generally acknowledged that the explanation of these theories in textbooks and in actual practice is usually inadequate. Problems connected with these theories are often treated superficially or in passing, with the result that there exists an uncertainty as to how to apply these methods in a practical case.

It is particularly true that the limitations of the se techniques are very seldom pointed out; however, if properly applied they can indeed be utilized to analyze certain very complex phenomena and are of considerable help in setting up experiments. At one extreme, therefore, similarity theory is sometimes considered infallible, while at the other it is expected to give only trivial results. Actually dimensional analysis is in its own right a science that requires substantial study and experience to be applied with full confidence. Over the last

several decades many specialized texts ${ }^{\dagger}$ on this subject have appeared, $(2-10)$ of which the most recent and most relevant appears to be Reference 3 .

Very briefly, the basis of dimensional analysis rests with the fact that every physical relation between the regular dimensional quantities can be formulated as a relation between nondimensional quantities. It is important that by this step the number of the original dimensional quantitites is automatically reduced to a smaller number of dimensionless parameters. All the nondimensional variables of the phenomenon can then be considered as a function of these

*Dimensional analysis and similarity theory define the dimensionless parameters which represent the fundamental relations and processes and determine the conditions under which model experiments are to be performed.

tHersey (Reference 1) has compiled a list of 20 books and refers to countless articles written on the subject matter of dimensional analys is. 
independent nondimensional parameters. ${ }^{*}$ According to Sedow ${ }^{(4)}$ the general procedure is as follows: "We start every study of mechanical phenomena with a survey, picking out the basic factors which define the quantities of interest to us and in the broadest sense drawing on already familiar examples of phenomena to construct a model of the processes under investigation. A sound survey is very often a difficult problem which requires, on the part of the investigator, a great deal of experience, intuition and a preliminary qualitative explanation of the mechanism of the process being studied."

This literal quotation clearly points out that it is extremely difficult to give a straightforward prescription for arriving at useful similarity parameters and model testing procedures. $\dagger$

Therefore, in tests involving liquid metals and water any new application of the similarity law should be investigated thoroughly before any firm conclusions are drawn from model testing with water.

As a practical example of using dimensional analysis in a new but very complex liquid metal flow problem the case of the two-phase flow stability study for the SNAP 50/SPUR liquid metal boiler may be cited. (11) These investigators specifically stated that they used their own previous experience and intuition to find the right dimensionless parameters.

However, there is one other way to arrive at the important dimensionless similarity parameters. This approach ${ }^{\S}$ uses strict mathematical arguments in reducing the pertinent equations to a dimensionless form. In this way all intuition and guesswork is eliminated. But this procedure is limited only to those few cases where a good theory in manageable mathematical form is available (as e.g., in the case of the Navier-Stokes equations which lead to the Re-number concept).

*For details and mathematical proof see, e.g., References 5-7.

$\dagger$ As an example of this uncertainty the case of the formulation of a similarity law for heat transfer may be cited. (4) It took several decades before a certain questionable argument was resolved, with such well-known scientists as Raleigh (UK), Riabouchinsky (France), Bridgman (USA), Sedow (USSR) participating in the discussion.

$\S$ This method is sometimes called differential similarity $(12)$ because it usually involves the consideration of the underlying differential equations. 


\section{PROPERTY DIFFERENCES BETWEEN WATER AND LIQUID METALS}

Basically it is possible to derive all macroscopic physical properties (including transport properties) of a liquid from its microscopic characteristics such as molecular (or atomic) structure and intermolecular potential. Efforts have been made $(13-19)$ and are still being vigorously pursued at the present, particularly in the case of water ${ }^{(20,21)}$ and of liquid metals, ${ }^{(22-24)}$ to shed some light on these relationships. However, the subject is very difficult and complex and at this time no convincing and comprehensive theory exists for relating the intramolecular forces to the bulk macroscopic properties. (25) In those few cases where properties have been theoretically predicted on a quantitative basis, they are often not very accurate since a number of simplifying assumptions must be made to make the mathematical formulation and solution of the theory more tractable. Therefore, our best present knowledge of the properties of liquids is still based exclusively on experimental measurements.

A compilation of the properties of liquid sodium (and to a lesser extent potassium and $\mathrm{NaK}$ ) can be found in References 26 to 28. A new edition of the Liquid Metals Handbook ${ }^{(26)}$ is being prepared and is scheduled for publication in 1968. The properties of water have been compiled in Reference 29.

The properties of liquid metals and water are quite similar in fluid flow behavior; however, they are very different in thermodynamic behavior. In particular, the density $(\rho)$ of liquid sodium at $1200^{\circ} \mathrm{F}$ can (for most practical purposes) be assumed to be $0.8 \mathrm{~g} / \mathrm{cm}^{3}$, which is $80 \%$ of the density of water. It does not change very much with temperature. The dynamic viscosity $(\eta)$ of sodium at $1200^{\circ} \mathrm{F}$ is about $20 \%$ of that of water at $68^{\circ} \mathrm{F}$ (see Figure 1). Compared with the wide range (approximately $10^{4}: 1$ ) of viscosities which exists in the realm of liquids, water and sodium are relatively close. Therefore, in many cases where viscosities are only of secondary importance (as in the overall evaluation of large pumps), such a discrepancy as a factor of 5 can often be disregarded. However, in other cases where viscosity is of primary importance (e.g., in the determination of friction factors, etc.), a factor of 5 is too large to be ignored. It is possible, of course, to decrease the discrepancy in the viscosities by heating the water to higher temperatures. In order

LMEC $-68-4$ 


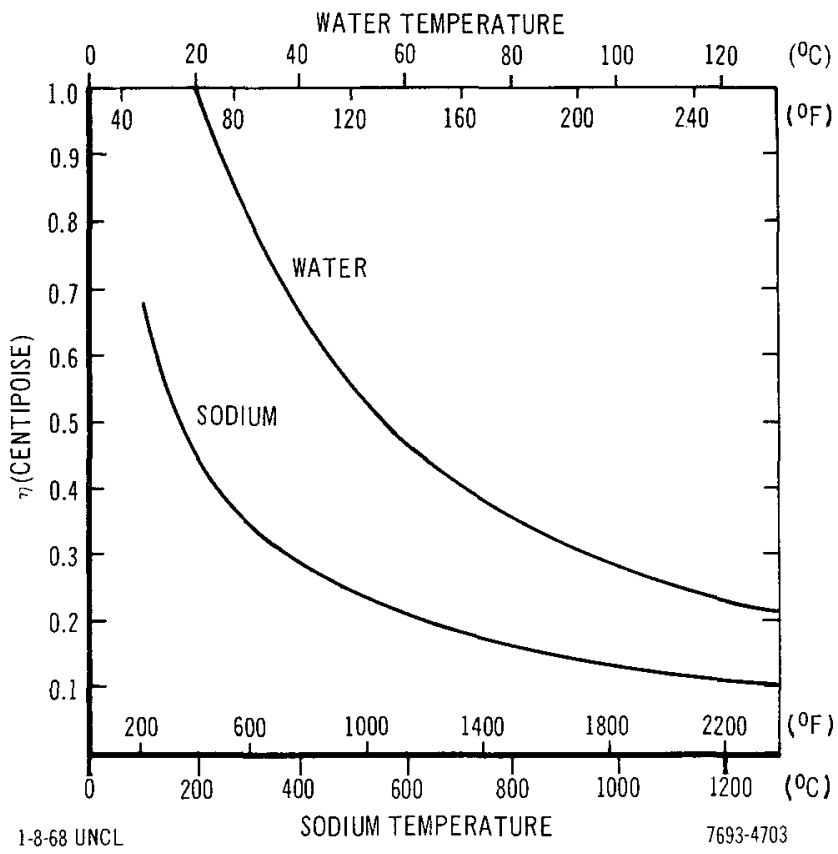

Figure 1. Dynamic Viscosity of Water and of Liquid Sodium

to equal the dynamic viscosity $(\eta)$ of liquid sodium at $1200^{\circ} \mathrm{F}$ it is necessary, however, to heat the water to about $290^{\circ} \mathrm{F}$, which is well above the atmospheric boiling point of water and would require a pressurized system good for at least 60 psi.

The kinematic viscosities $(\nu)$ of water $\left(\right.$ at $\left.68^{\circ} \mathrm{F}\right)$ and liquid sodium $\left(1200^{\circ} \mathrm{F}\right)$ differ by a factor of 4 (see Figure 2). Again it is possible to decrease this dis crepancy by heating the water to high temperatures so that finally at about $250^{\circ} \mathrm{F}$ the kinematic viscosity of water equals that of $1200^{\circ} \mathrm{F}$ sodium. For many (but certainly not all) practical purposes, however, heating to higher temperatures is unnecessary; it is usually sufficient to simulate a suitable nondimensional parameter (such as the Re-number) which contains the viscosity merely as one of several independent factors. Nevertheless it must be pointed out that it is not possible to simulate both the dynamic and the kinematic viscosities of water and sodium at the same time. This means that water can never be a perfect fluid medium for simulating liquid metal flows in a model test. However, the errors that would be introduced due to this mismatch between dynamic and kinematic viscosities are relatively small and can often be ignored altogether. 


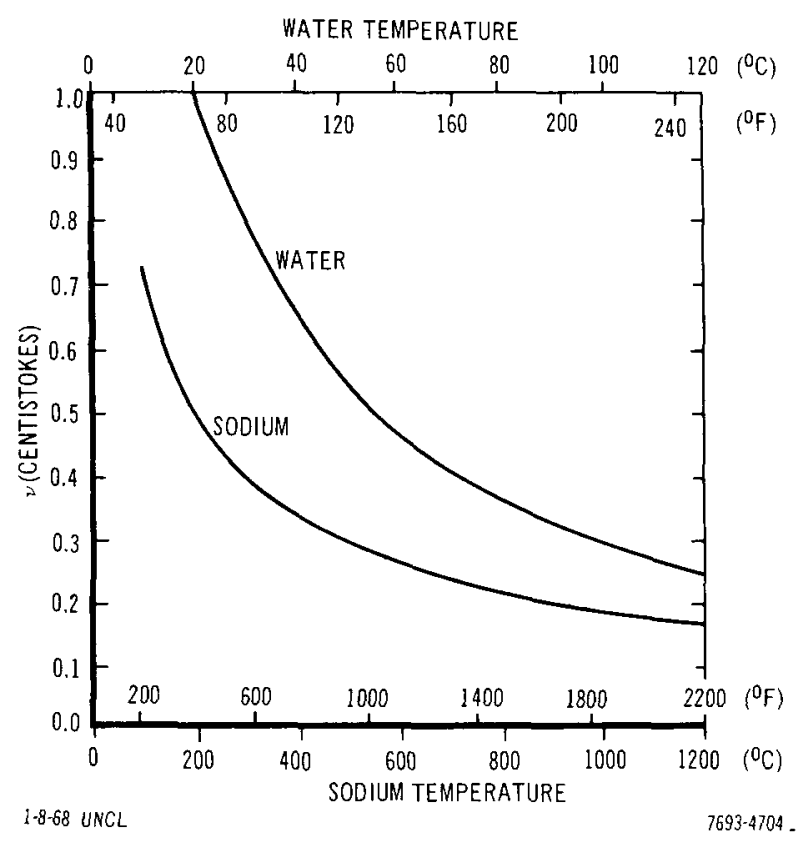

Figure 2. Kinematic Viscosity of $\mathrm{W}$ ater and Liquid Sodium

There are some additional properties (such as heat conductivity, vapor pressure, compressibility etc.) which are different for water and for liquid metal. But these are important only for certain types of applications and accordingly they will be discussed under the appropriate subject headings.

A substance that at room temperature $\left(65^{\circ} \mathrm{F}\right)$ has practically the same viscosity and density as liquid sodium at $1200^{\circ} \mathrm{F}$ is acetaldehyde $\left(\mathrm{CH}_{3}-\mathrm{CHO}\right)$, a relatively inexpensive organic clear liquid. ${ }^{(30)}$ Strictly from the viewpoint of fluid mechanics this substance is far better suited for simulating liquid sodium flows than water at ambient temperature. A disadvantage might be its flamability and its rather low boiling temperature $\left(69^{\circ} \mathrm{F}\right)$. $^{*}$ Unless used in a pressurized system it would easily tend to cavitate.

So far no systematic search is known to have been performed to uncover the most suitable modeling fluid with respect to liquid metal applications.

Because of its very low viscosity hot liquid metal flow actually resembles potential flow more closely than that of almost any other fluid. Potential flow, which is based on the assumption of a frictionless fluid, can in certain cases be treated theoretically quite well by powerful mathematical methods (i.e., potential theory, conformal mapping, etc.). $(32,33)$

*On the other hand, acetaldehyde should not cause rust problems (common with water) of the type encountered with water model testing for EBR II.(3 1)

LMEC -68-4 


\section{MODEL TESTING OF FLOW IN PIPES, REACTOR CORES, AND SIMILAR GEOMETRIES}

Model testing is used for almost all major flow problems encountered in nuclear engineering and is by no means restricted to a particular need for liquid metal testing. For example, flow problems in water-cooled reactors usually have to be investigated by scaled-down model tests. The use of liquid metals in reactors only compounds the difficulties of a situation that is already quite complex.

The basic reason for this condition is the rather incomplete state of our present knowledge of the fluid mechanics of internal flow. (34) These flows are highly complex because their bounding surfaces are often of complicated geometry, mechanical and thermal energy is frequently exchanged with the fluid passing within them, and the majority of the configurations are dominated by turbulent shear flow.

Because it is unlikely that this situation will improve substantially in the near future, model testing will undoubtedly continue as the main instrument for dealing with fluid flow problems encountered in the design of new types of nuclear reactors. (35) It is, therefore, also not surprising that the majority of the quantitative relations used in internal flow problems are not really true equations, derived in an orderly fashion from basic laws, but merely empirical (or sometimes semi-empirical) correlations which have been found in extensive experiments and tests. Here dimensional analysis is often very helpful in reducing a multitude of test data to more easily manageable relationships between significant nondimensional parameters. As previously mentioned, however, one can never be completely confident that the correct choice has been made in selecting these particular quantities unless some support from differential similarity exists .

Probably the most successful and most widely known application of dimensional analysis is the case of established pipe flow, which is characterized by the nondimensional parameter $\mathrm{Re}=\rho \mathrm{ud} / \mu$, known as the Reynolds number; ( $\rho$ and $\mu$ are the density and the viscosity of the fluid respectively, and $u$ is its average velocity in the pipe of diameter d). Established flow shall mean here,

*For a derivation see Reference 36. 
that the flow is Newtonian, incompressible, isothermal, and steady, and that end effects of the straight and smooth pipe can be neglected. Within the framework of these as sumptions it turns out that the pressure drop $\Delta p^{*}$ [made dimensionless ${ }^{\dagger}$ by dividing through the dynamic pressure $\left.(\rho / 2) u^{2}\right]^{\S}$ is a function of Re only:

$$
\frac{\Delta p}{(\rho / 2) u^{2}}=f_{1}(R e)
$$

Dimensionless analysis makes no predictions as to the specific functional relationship. In fact, the function $f_{1}$ turns out to be different for the laminar and for the turbulent case. The dividing line between these two cases is defined by the so-called critical Re-number $(\approx 2000)$. Since the Re-number makes no distinction as to the kind of fluid (as long as it is a Newtonian fluid), pressure drop coefficients a re equal as long as the kinematic viscosity $\nu=\mu / \rho$ of the fluids is the same. Therefore, in established pipe flow, e.g., liquid sodium of $300^{\circ} \mathrm{F}$ can be simulated by water of $115^{\circ} \mathrm{F}$. By juggling the values of $\mathrm{u}, \mathrm{d}$, and $\nu$, meaningful model testing can also be done with scaled-down (or scaled-up as the case may be) versions of the pipe as long as Re stays constant. ** Within the scope of this reasoning it is therefore also pos sible to use even gases (such as air) as a modeling fluid. (38)

\footnotetext{
${ }^{*} \Delta \mathrm{p}$ is the difference of the static pressures (wall pressures), measured at separate stations along the pipe. It has been shown that the accuracy of measurements of static pressures through flush holes in the wall is affected by the size of the hole and the viscosity of the fluid. Generally, the accuracy increases with decreasing hole diameter and increasing viscosity. This has some implications if one is to compare experimental data obtained in water and in liquid metal tests. As resulting errors are generally quite small (on the order of 1 percent) the mere mentioning of the effect might suffice here.

$\dagger$ The dimensionless parameter $\Delta p / \rho u^{2}$, is sometimescalled the Euler-number (Eu).

$\S$ The dynamic pressure $(\rho / 2) \mathrm{u}^{2}$ often serves as an alternate measure for fluid flow velocity $u$. It corresponds to a fluid column of the height h so that $(\rho / 2) \mathrm{u}^{2}=\rho g h$ or $\mathrm{h}=\mathrm{u}^{2} / 2 \mathrm{~g}$, where $\mathrm{h}$ is called the velocity head, while $g$ is the constant of gravity. As the density cancels out in the above formula, the nomogram in Figure 3 can be used for water as well as for liquid metals. It must be remembered that 1 in. of water corresponds to $0.0361 \mathrm{psi}$ (or $5.20 \mathrm{lb} / \mathrm{ft}^{2}$ ) and that 1 in. of liquid sodium (at $1200^{\circ} \mathrm{F}$ ) corresponds to $0.0288 \mathrm{psi}$ (or $4.16 \mathrm{lb} / \mathrm{ft}^{2}$ ).

**The most successful application of Re-number similarity can be found in aerodynamics where the flow a round bodies (e.g., airplanes) is usually simulated in wind tunnels. However, in liquid metal technology there is very little opportunity for this type of testing which usually implies a test object within an infinite body of fluid. Liquid metal flows are practically always bound by some kind of rigid walls. Admittedly, the test section in wind tunnels is also bounded by walls, but their influence is usually accounted for in rather complicated wall corrections. (37) Liquid metal flows almost always fall into the large category of internal flows. (34)
} 


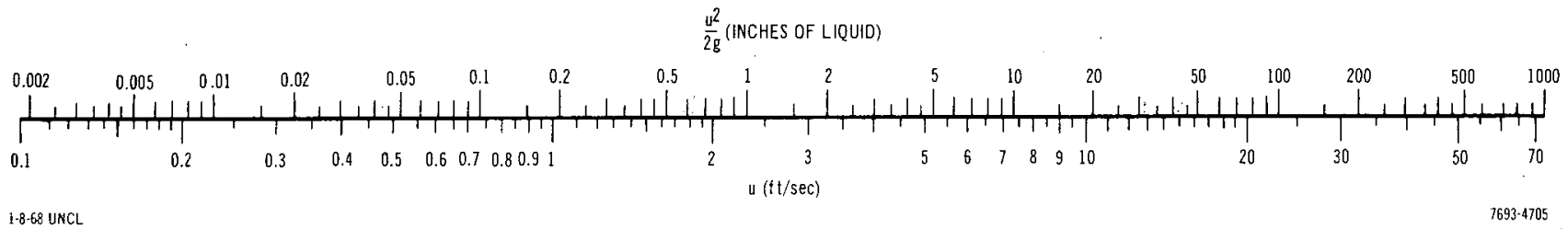

Figure 3. Dynamic Pressure (Velocity Head) as a Function of Velocity

But this is also as far as this simple form of similitude goes. For example, when dealing with a pipe that has a rough inner surface (as most industrial pipes $\mathrm{do}$ ), an additional parameter $\mathrm{k} / \mathrm{d}$ enters (where $\mathrm{k}$ is the average height of a roughness element) and now the functional relationship takes the form

$$
\frac{\Delta p}{(\rho / 2) u^{2}}=f_{2}(R e, k / d)
$$

For the specific liquid metal case under investigation this means that in order to simulate liquid metal flow by water in a rough pipe one has to duplicate the roughness ratio as well. This can most reliably be done by full-scale test because it would be very difficult (if not impossible) to scale down the geometric roughness contours of a commercial pipe.

It should be emphasized at this point that dimensional analysis does not include anything about the relative importance of the two parameters $\mathrm{Re}$ and $\mathrm{k} / \mathrm{d}$. Such information must be found either from exact theory (which is too difficult and not feasible yet) or from experiments. As regards pipe flow it turns out that for low Re-numbers (in the laminar and transitional regime) the Re-number is clearly the dominating parameter, whereas with higher Renumbers (in the turbulent regime) the roughness parameter becomes more and more important, so that for $\operatorname{Re} \widetilde{\sim} 10^{6}$ the Re-number influence is usually negligible.

This has particular significance for liquid metal testing. It means that at very high Re-numbers ${ }^{*}$ it is more important that flow tests be performed with

*Anticipated Re-numbers in typical full-scale pipes, e.g., those in the Sodium Pump Test Facility (SPTF) are on the order of $10^{7}$. 
full-scale pipe samples than with a fluid that has the same Re-number." (This presupposes that the liquid metal is wetting the pipe perfectly, i.e., its temperature is high enough.) It must be kept in mind that over the years the roughness of the pipes (or rods) may change due to continued corrosive action of the hot sodium. As a result the originally determined pressure drop for a pipe section might change also. Increased hydraulic resistance (about $10 \%$ ) to the coolant flow in the core of an operating reactor has been observed in the case of a water cooled reactor and has been reported in the literature. (39)

Again, by reasoning derived from dimensional analysis, it can be argued that the pressure drop for a bent pipe (curved duct) would be of the following form: $†$

$$
\frac{\Delta p}{(\rho / 2) u^{2}}=f_{3}(R e, k / d, d / R, \delta),
$$

where $R$ is the radius of curvature of the bend and $\delta$ its total angle. Here too, dimensional analysis contains nothing relating to the specific form of the functional relation, which again can only be determined empirically. Because end effects are still not being considered, the straight ends before and after the curved section should be long enough for established flow to exist at both ends. If experimental data are to be correlated for pressure drop around a curved duct they should be taken between points of established flow. If this is not done with care there will usually be an increase in the scatter for the correlated data. This example also makes clear that with an increasing number of independent dimensionless parameters it becomes more and more difficult to generate experimental conditions which are truly similar in the sense of dimensionless analysis. In the case of curved pipes the data reported in the literature are much more scarce and do not correlate as well as for straight pipes. $(40,41)$

*Conditions could be different for flow between fuel rods $\left(\operatorname{Re} \approx 10^{5}\right)$, depending on the smoothness of the cladding.

tIt is not likely that these four parameters constitute all that are of importance in curved pipe flows. There is certainly an influence of centrifugal and Coriolis forces which set up secondary flows. These forces are proportional to the density $\rho$ of the fluid. Therefore such forces could be disregarded when substituting water tests for liquid metal tests, only if one as sumes that $\rho_{\mathrm{Na}} \approx \rho_{\mathrm{W}}$. This is true within about $20 \%$, as shown on page 13 . 
A significant point to be remembered always for model testing is that for large Re-numbers the accurate simulation of the geometry of the curved pipe section is more important than Re-number simulation. (42) Under such circumstances, then, it does not matter too much whether such full-scale tests are performed with liquid metal or with water. If one deviates, however, from the conditions for which this similarity is valid, deviations in results must be expected.

As mentioned before, Reynold's similarity law for viscous flows can be derived also in a purely mathematical way from the basic Navier-Stokes equations. ' All restricting as sumptions to be used in its derivation are, therefore, also applicable to Re-number similarity analysis; (e.g. , Newtonian, stationary, incompressible fluid without a free surface and without external forces, etc.). Particular attention is invited to the neglecting of all mass forces which are the result of gravity, centrifugal and/or Coriolis action. Consequently, flows in curved ducts and with a free surface are eliminated from a strict application of the similarity law. If a free surface is involved an additional dimensionless parameter, the Froude number $F=u / \sqrt{g h}$ ( $u$ being the velocity and $h$ being the depth of the fluid, $g$ is the gravitational constant) has to be observed for similarity. No such additional parameters have been derived to account for centrifugal and Coriolis forces.

Flows with a free surface are quite rare in liquid metal applications. Consequently, Froude analysis is rather unimportant in this field. Since the fluid in surge tanks or in pumps does not have a net translational velocity it is not of real interest as far as loop performance is concerned; there are some transient problems (such as vortexing while draining, etc.) which are not subject to similarity analysis either and they will be treated separately on page 81 .

Centrifugal and Coriolis forces are usually the cause of the generation of secondary flows which make the flow patterns very complicated and unpredictable. $(44,45)$. There may be many problems of this sort in a liquid metal loop

*As previously mentioned, the effects of non-established flows (e.g., insufficient pipe length ahead of and behind the curved section), effects of centrifugal forces, heat transfer, transients, roughness, etc., all must be negligibly small.

†Only the results are presented here. For details see, e.g., Reference 43. 
because a closed and compact loop necessarily must have a certain number of elbows, tees, etc. Therefore the lack of a similarity law for these cases is quite unfortunate. Luckily however, the density of water is quite close to that of sodium (within $20 \%$ ) so that model testing in water is at least a good qualitative approximation. For higher accuracy requirements a fluid like acetaldehyde I (see page 15) should be used instead of water.

Another conclusion drawn from the mathematical deduction of Re-number analysis is that the boundary conditions for the flows must be similar. This is fairly self evident as far as the geometrical shape of the flow contours (e.g., shape of an immersed body) is concerned, but it also implies that the incoming flow pattern is similar. In the wind tunnel testing technique great care is usually taken to make the flow entering the test section parallel and very uniform (with low free-stream turbulence). However, in hydraulic testing of flow in conduits it is often overlooked that in order to have similarity, the flow entering a certain component under test must have similar velocity distributions for the two cases which are to be compared under the similarity rules. Specifically, for testing of components to be used in a liquid metal loop, it means that in water (model) testing the incoming flow must be similar to the conditions expected in the real case. This is best done by also simulating the flow (and conduit) upstream of this particular component. If it happens that this can be done by a long section of straight pipe or by a plenum, this would then appear to be a good simulation. However, as is likely to happen, if the upstream portion contains an elbow (or another component that disturbs uniform flow distribution) this part of the loop should be attached to the component under test.

The full consequences of this requirement become clear if it is realized. that the flow into this section of the loop (that attached upstream of the component under test) is in turn influenced by sections still further upstream. This type of reasoning can ultimately be applied all the way around the loop.

The following, then, appears to be the real crux of model testing in liquid metal applications: depending on the particular design it is easily possible that there is no section in the whole loop in which a uniform established flow condition exists. The flow in each section or component is influenced by the section (or component) further upstream, consecutively all the way around the loop. Truly 
quantitative results can be obtained in such a case only from the configuration as a whole.

On the other hand, it should not be concluded that approximate answers (in some cases even fairly good ones) could not be obtained by water model testing. An estimate of the size of errors that might be incurred in each particular case is rathe $r$ difficult, however, because of the interaction of so many causes contributing to this error. By the same token, it is practically impossible to give one fixed figure for the error incurred when water model tests are applied to liquid metal flows. As can be implied from what has been said previously, this figure necessarily would depend on many details which a re characteristic for a particular test case only.

Further study and actual experience gained in comparing liquid metal data with those obtained from water tests will enable us to make more precise error estimates. Unfortunately very little quantitative information on this subject is available from the current literature.*

Statements found in the literature $e^{(47-50)}$ relating to the hydraulic similarity between liquid metals and water are usually couched in rather general terms and are often superficial and misleading. Discussion in this treatise has shown that it is by no means always sufficient for Re-numbers to be duplicated for hydraulic testing, as is frequently implied. It is also not true that it is very simple to make corrections for lesser effects. A typical example of an incorrect statement, found in Reference 51, is: ". . if full scale geometry and fluid velocity are duplicated, and also if the kinematic viscosity $\nu=\mu / \rho$ are being made equal for liquid metal and for water, then the velocity heads $(\rho / 2) u^{2}$ are alsoduplicated." This is impossible because the densities of liquid metal and water are unequal. Furthermore, in the same context, it makes no sense at all to form a Froude number by using the equivalent diameter. It is equally wrong to form a Froude number with the pipe length as is done in Reference 52. (It has been shown in

*Whereas a considerable amount of data $(41,46)$ exists for estimating the hydraulic resistance in various simple flow configurations (which can undoubtedly be used for many practical design purposes), this information is often not accurate and reliable enough to detect those relatively small differences which might be due to secondary flow effects in the case of liquid metals, even if the corresponding liquid metal data were available. 
this document that the usefulness of the Froude number similitude is also quite limited for liquid metal applications.)

A survey of recent water testing for reactor design purposes shows that water model tests have been used regularly for exploring the flow inside the reactor vessel, both for water-cooled $(31,53,54)$ and liquid-metal-cooled reactors. $(31,48,55)$ In the following text, a critical review is given with regards to the purpose of such tests, their implementation, and their usefulness.

For economic reasons (the most important for carrying out model tests) there is usually a scale-down of the geometric dimensions (for an exception see References 56 and 57). The higher the scale-down, the more errors to be expected. As explained in more detail in other parts of this report, errors might be generated by boundary layer effects which become more pronounced at smaller dimensions. Therefore, the scale factor is usually a compromise between economic and technical viewpoints. Linear scale-down ratios in the order of $1: 4$ to $1: 10$ are most frequently used.

Sometimes it is rather expensive to simulate every minute detail in its exact geometric shape and proportions; exact simulation of the many internal parts of a reactor such as fuel rods, etc., would require a considerable amount of work for the model shop. Therefore, attempts have been made (with apparent success) to simulate certain intricate components with devices that have the same net effect on the flow. For example, the pressure drop through a fuel subassembly has been simulated with a series of screens of equal resistance. Estimating the errors generated by introducing substitute devices is very difficult and will depend on the particulars of each case. While in one instance the errors may be negligibly small, in another the flow downstream of the substitute device may turn out to be completely different (e.g., the flow distribution generated by screens and by a piece of straight pipe of equal resistance).

So far error estimates havebeen conspicuously lacking in the literature on model testing for nuclear reactor applications." However, these estimates should be attempted at least, otherwise an uninitiated reader might take the test data at their face value.

*Judging from the experience gained in wind-tunnel model testing ${ }^{(37)}$ (where error estimating is by now a highly developed technique) it probably will be a long time before reliable error estimates can be expected. 
A few remarks concerning the concept of hydraulic diameter are in place here. Due to secondary flow effects in pipes of other than circular cross section, there is usually a more complicated (a three-dimensional instead of a simple one-dimensional) flow distribution in such channels. (42) Depending on the magnitude of this effect the pressure drop can be slightly different from one being determined by use of the hydraulic diameter and the regular friction factor. (58) These small secondary effects apparently change somewhat with Re-number, with the density of the fluid and the roughness of the pipe channel, and also with the geometrical shape of the channel itself.

In nuclear applications such channels are frequently incurred for flow between fuel rods. It is to be expected, therefore, that water tests will not simulate liquid metal flows too closely for such configurations. However, average errors are probably less than $10 \%$.

As previously mentioned, some sort of model tests have almost always been performed during the design and development stages of practically any new reactor (Ref's. 31,47-49,53,59-70). Generally, the aim of such tests has been the investigation of the internal flow pattern in reactor cores (flow through subassemblies), plenum chambers, vessels, etc. Furthermore, the fluid flow effect of a particular geometrical shape of a flow duct, of guide vanes, orifices, and other details (too numerous to mention) have been the subject of model testing. Pressure drop measurements and mixing studies for reactor cores, heat exchangers, steam generators, etc., have also been performed by water model tests. $(64,65,71,72)$ Models of various scales (both scaled-up and scaled-down) have been used. Very often these model tests have not been very elaborate or sophisticated, but nevertheless they usually served their purpose very well. In some cases, however, it appears doubtful whether the tests really yielded the information and the results they were intended for. In such cases the description and the reasoning for the specific purpose of a model test often reflect some misconceptions as to its usefulness and the interpretation of the resulting test data.

Hydraulic water model testing of reactor cores (and incidentally also water testing of heat exchangers) ignores the heat transfer that occurs in actuality. Although it has been shown that the overall temperature effect on fluid flow is

\section{LMEC $-68-4$}


small, at least for moderately low, heat transfer rates, ${ }^{(51)}$ effects on boundary layers can be quite significant.

However, with increasing heat transfer rates the interaction of the hydrodynamic flow pattern with the heat flow pattern becomes more and more important. This is compounded even further as long as water is used to simulate liquid metal flow. For example, it is immediately obvious that in a water model (under isothermal conditions) the density and viscosity of the water are constant whereas in the corresponding prototype sodium-cooled reactor conditions are never isothermal, so that temperature and hence density and viscosity depend on geometrical position. A more detailed discussion of such problems can be found in Section VIII on heat transfer modeling.

Another argument frequently cited in favor of water testing as compared to liquid metal testing is that transducers for measuring water flow are commercially a vailable in a wide variety and are generally quite accurate. This is usually (at least up to the present time) not the case for liquid metal transducers. Why then, the argument goes, should tests be made with liquid metals if the measured data are not sufficiently accurate. But there seem to be no cogent reasons (given time and money) why accurate transducers could not be developed for service in hot liquid metals. This is a field that should be pursued now with intensified effort.

At the present time, water model data stands by itself. (31) Reactors are not instrumented extensively enough to check predictions based on model behavior. If confidence in model testing is to be increased, and model testing techniques improved, verification (at least in a few cases) of the quantitative details derived from water tests is required.

\section{SUMMARY ON PIPE F LOW}

Whereas liquid metal flows in straight smooth pipes can easily and correctly (within about $2 \%$ ) be predicted theoretically, they can also be simulated accurately by water model tests. If the effect of roughness is to be tested, this has to be done with a prototypical pipe. Curved ducts and other flow irregularities (such as tees, constrictions, enlargements, etc.) can be simulated in water 
only with somewhat lower accuracy, because of secondary flow effects. It is often not possible to reliably test single components of a liquid metal loop in an isolated way unless the effects of upstream disturbances a re also properly simulated. Quite generally, accuracy of water model testing depends on how closely all corresponding liquid metal flow conditions are actually being simulated in detail. With special care and by using a low viscosity liquid such as acetaldehyde in a full-scale model, accuracy should approach about a few percent. 


\section{MODEL TESTING OF PUMPS}

It is apparent that the reasoning that merely Re-numbers need be duplicated to simulate liquid metal flows by water tests is certainly not valid for pumps. This is so because external forces enter into the flow equations for pumps, which thus make them more complicated. Therefore, dimensional analysis must be used to find a complete appropriate set of dimensionless parameters which are applicable to the fluid mechanics of pumps.

It can be shown ${ }^{(73)}$ that the efficiency $\eta$, the head $H$ of the liquid (either sodium or water); and the shaft power $P$ (when written in dimensionless form) are dependent on the same group of dimensionless similarity parameters:

$$
\begin{aligned}
& \begin{array}{l}
\text { Head coefficient } \\
\begin{array}{l}
\text { (also called energy } \\
\text { transfer coefficient) }
\end{array}
\end{array} \quad \psi=\frac{\mathrm{gH}}{(\mathrm{ND})^{2}}=\mathrm{f}_{1}\left(\frac{\mathrm{Q}}{\mathrm{ND}^{3}}, \frac{\rho \mathrm{ND}^{2}}{\mu}, \text { geometry ratios }\right) \\
& \text { Efficiency } \\
& n=f_{2}\left(\frac{\mathrm{Q}}{\mathrm{ND}^{3}}, \frac{\rho \mathrm{ND}^{2}}{\mu} \text {, geometry ratios }\right) \\
& \text { Power coefficient } \\
& \widehat{P} \equiv \frac{P}{\rho N^{3} D^{5}}=f_{3}\left(\frac{Q}{N D^{3}}, \frac{\rho N D^{2}}{\mu}, \text { geometry ratios }\right)
\end{aligned}
$$

where $\mathrm{N}$ is the speed of rotation, $\mathrm{D}$ is a characteristic diameter, such as the impeller diameter, $Q$ is the volume flowrate, and $\rho$ and $\mu$ are the density and the viscosity of the pumped fluid. The geometry ratios are the dimensions of the pump (made dimensionless by divisions with $D$ ) which characterize its internal shape.

It can be seen that the parameter $\rho \mathrm{ND}^{2} / \mu=N D^{2} / \nu$ has the form of a $\mathrm{Re}-$ number, which is actually indicative of the order of magnitude of the Re-number for the flow inside the pump. (It is possible to form other Re-numbers for specific portions of the flow inside the pump, but this is necessary only if the flow pattern is considered in detail.) Fortunately, for the large sodium pumps (such as those to be tested in the SPTF) the design Re-numbers appear quite large (on the order of $>10^{7}$ ). At such a magnitude the effect of Re-number on the flow parameters becomes rather small and can be neglected in a first approximation. 
Experiments with water have confirmed these assumptions, and at this time it must be assumed that they hold true for liquid metals also; at least there are no known reasons why they shouldn't. With these assumptions the functional relationships for geometrically similar pumps (with no difference for water or for liquid metal pumps) become simply:

$$
\psi=\mathrm{f}_{4}(\varphi) ; \eta=\mathrm{f}_{5}(\varphi) ; \hat{\mathrm{P}}=\mathrm{f}_{6}(\varphi)
$$

This means that the flow parameter $\varphi=\left(Q / N D^{3}\right)$ is the only coefficient that determines all the others. The actual form of the functions $f_{4}, f_{5}, f_{6}$ must be determined by experiments. Therefore, within the framework of the preceding assumptions, a liquid metal pump can be developed first as a water model (as a scaled-down model if this is desirable; however, it must be remembered that pump Re-numbers decrease with the square of the diameter, and that errors might increase substantially if Re-numbers are allowed to drop too low). From the dimensionless coefficients obtained in water tests, the actual corresponding pump performance parameters for liquid metals can then easily be determined. If cavitation occurs within the pump, this procedure breaks down."

The usefulness of this similarity law for pumps has been demonstrated to a certain extent by the successful development of liquid metal pumps for various liquid-metal-cooled reactor projects. $(56,74-76)$ However, a statement in the literature, (77) "The fluid mechanics of liquid metal pumps are completely predictable on the basis of experience with water and conversion based on a knowledge of the physical properties of the liquid to be pumped," seems to be an overstatement since apparently the various limitations of the similarity law have not been considered. (The author would agreee with such a statement. if the word "completely" were replaced by "almost completely.")

Pumps are frequently designed and developed under the assumption of frictionless flow. If such a pump is tested in cold water, for instance, it will show an efficiency $\eta$ which is smaller than one, because there are always friction losses in the pump which are unavoidable. As the viscosity of liquid sodium at $1200^{\circ} \mathrm{F}$ is about five times smaller than that of cold water, it is expected that

*Additional remarks on cavitation on page $31 \mathrm{ff}$. 
the efficiency of the same pump would be higher for liquid metal operation. This assumption was corroborated by experience with the Hallam Nuclear Power Facility (HNPF) reactor ${ }^{(78,79)}$ where it was only necessary to operate the pumps at approximately $80 \%$ of design speed to deliver the required flow.

Consequently, if a new pump design is verified in water tests, such tests can usually be considered conservative for liquid metal applications. In fact, it should be possible to design a pump specifically for given liquid metal requirements, and such a pump should be somewhat smaller than an equivalent pump designed for corresponding water requirements (provided that cavitation can be avoided).

For a more accurate treatment of pumps ${ }^{(80)}$ it must be remembered that in a real fluid (such as water or liquid metal) similarity can exist only if the boundary layers, and in particular their displacement thicknesses, are similar; the local Re-numbers at comparable points must be the same. However, this requirement can be met only to a very limited extent in model testing if scaled models must be tested at Re-numbers smaller than those encountered in practice. As a result, the boundary layer on a model is thicker and the surface friction greater than would be obtained if true similarity existed. ${ }^{(81)}$ In many cases the differences are small and can be allowed for by an empirical correction during the conversion to the full-scale problem. In particular, the efficiency is usually higher than the value obtained from model tests. Nevertheless, there are cases in which flow at the higher Re-number deviates considerably from that at the lower one; scaled model testing is then useless. When this occurs the reason is usually that the boundary layer is laminar at the lower Re-number and turbulent at the higher one. Hence, a great deal of knowledge and experience are required to conduct accurate model tests so that valid conclusions can be drawn.

It has also been noted recently ${ }^{(82)}$ that boundary layers may interact strongly via secondary flows with the main flow in a rotating system (such as a pump), especially at high Re-numbers. Such little understood problems might arise with liquid metals in large pumps where high Re-numbers prevail. To investigate these problems experimentally it would be highly desirable to test in real liquid metals, or at least in a fluid of equally low viscosity, such as acetaldehyde. 
It is likely that for the large prototype liquid metal fast breeder reactors the pumps will not be operated at peak efficiency, especially if they must operate over a wide range of rotational speeds. Grindell ${ }^{(83)}$ and Eickhoff ${ }^{(56)}$ published some performance characteristics for liquid metal pumps and it can be seen that efficiencies generally peaked in the 65 to $70 \%$ bracket, which appears to be typical for the current generation of liquid metal pumps. It is probable that these efficiencies can be increased ${ }^{\dagger}$ considerably if more development effort were spent on hydrodynamic design details tailored especially to liquid metals. This might mean that effects of Re-number, boundary layers, surface roughness, cavitation would have to be considered (possibly even impellers with adjustable blade angles). If such secondary effects are to be tested in a model, this almost certainly would have to be done with the real fluid. It is therefore conceivable that the relatively simple proof testing of liquid metal pumps in the SPTF will gradually give way to more sophisticated development tests. It must be realized that higher efficiency $\$$ means savings not only in one prototype reactor, but in many future reactors of more or less the same type. Therefore, total savings could be quite substantial and a more refined pump development program could be economically justified.

Under the assumption that a pump with non-variable geometry (i.e., with fixed blade angles) operates at maximum efficiency, a new dimensionless parameter can be formed: the specific speed

$$
N_{S}=\frac{N(Q)^{1 / 2}}{(g H)^{3 / 4}} \text {. }
$$

*There is very little information available in the literature regarding the design procedure for liquid metal pumps. Also, there is hardly any mention of how performance predictions (based on design) were actually met in water tests and later with a real liquid metal fluid.

†The latest Byron Jackson Pump Co. estimate expects that the future large sodium pumps will be operated at about $84 \%$ efficiency. A pump designed for operation in the HNPF was reported to have reached a peak efficiency of about $95 \%$ in a sodium test loop (79)

\$aximim efficiency usually entails a minimization not only of operating expenses but also of size and cost of the whole reactor system. 
This parameter is of great importance in selecting the best type of pump for a given application. Here, the maximum efficiency condition replaces the condition of geometric similarity, so that any alteration in specific speed implies a change in pump design. In other words, each class of pump has an optimum efficiency within its own (fairly narrow) range of specific speed. Therefore, a pump for liquid metal applications can be selected (within the range of this approximation) purely for its general pump characteristics, ignoring initially any particular liquid metal peculiarities.

Again, if requirements call for higher accuracy (or closer tolerances) Renumber effects etc., must be accounted for, which would complicate matters considerably.

The cavitation limit mentioned in the preceding paragraphs usually represents an upper limit for the highest possible value of the specific speed of a pump, which means also, on the smallest possible pump size for given head and flow requirements.

Cavitation in a pump occurs with increasing speed, first at places of high local flow velocities where static pressures drop below the saturation pressure of the liquid. $(84,85)$ This incipient cavitation is characterized by the formation of small bubbles. When these bubbles are swept into regions of higher pressures, they collapse, generating very intense local pressure transients which can cause mechanical cavitation damage to any walls, blades, etc., which happen to be close by. Although it might be possible, in some cases, to accommodate a certain degree of water cavitation (especially supercavitation), it must be avoided in liquid metals under all circumstances. This is because any deleterious effect of hot liquid sodium $\left(>1100^{\circ} \mathrm{F}\right)$ on almost any type of material is intensified by cavitation.

The vapor (or saturation) pressure of $1200^{\circ} \mathrm{F}$ sodium is about 0.05 atm. Water has the same vapor pressure at about $100^{\circ} \mathrm{F}$. Therefore, to duplicate the vapor pressure of $1200^{\circ} \mathrm{F}$ sodium in a water test, water at $100^{\circ} \mathrm{F}$ should be used (see Figure 4 ). However, to duplicate the kinematic viscosity of $1200^{\circ} \mathrm{F}$ sodium in a water test, water at $250^{\circ} \mathrm{F}$ should be used. Clearly, it is impossible to satisfy both requirements simultaneously. A compromise solution is presented in the following text.

*Significantly, $\mathrm{N}_{s}$ is not dependent on $\mathrm{D}$. 


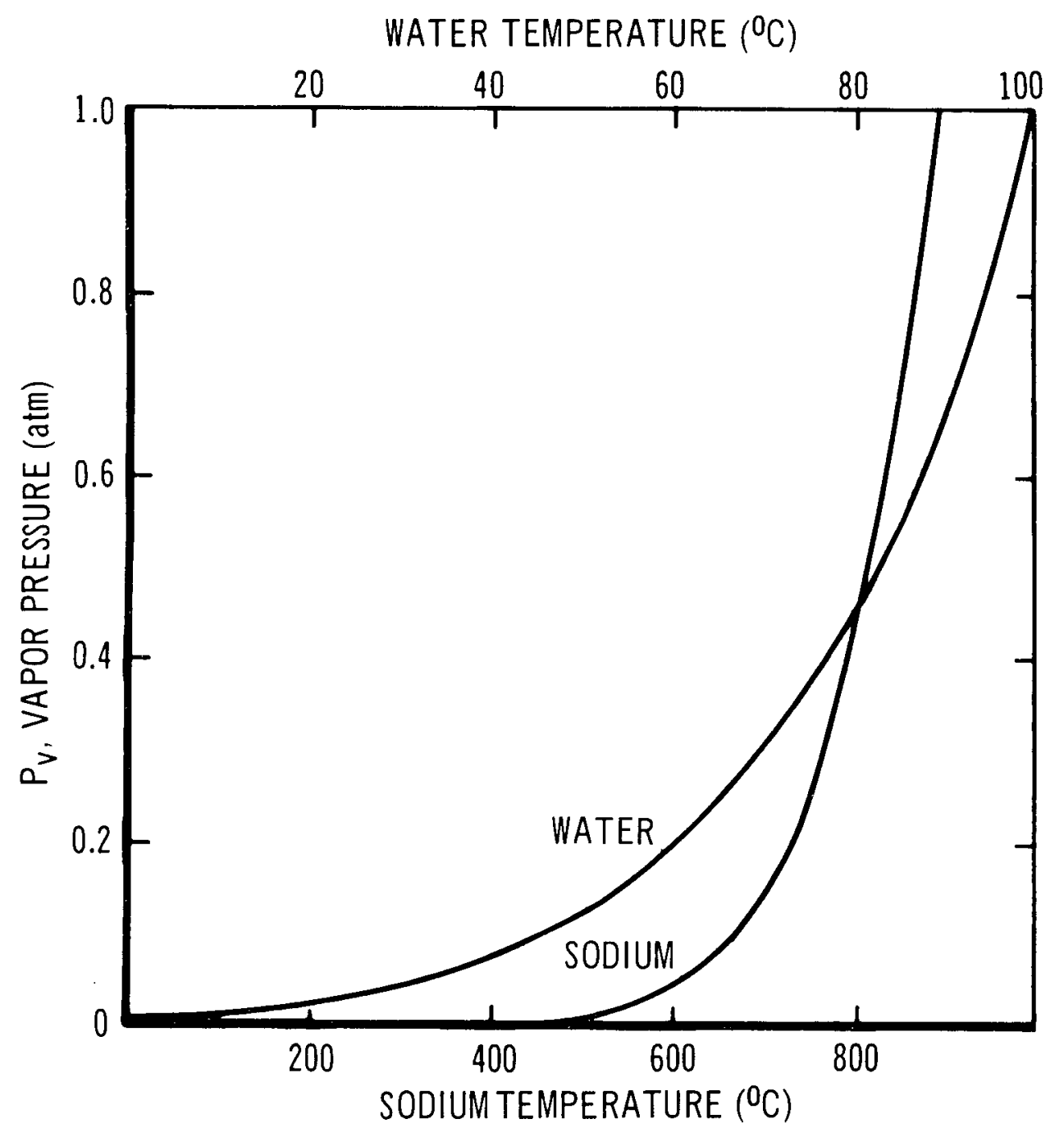

1-8-68 UNCL

7693-4706

Figure 4. Vapor Pressure of Water and of Liquid Sodium 
If, for example, the total pressure for a $1200^{\circ} \mathrm{F}$ sodium flow is 1 atm, the local static pressure will drop to $0.05 \mathrm{~atm}$ (which is equal to the vapor pressure) if the local velocity reaches about $50 \mathrm{ft} / \mathrm{sec}$; i.e., if $1 / 2 \rho u^{2}=0.95 \mathrm{~atm}$ (see Figure 3). In the case of a pump, the pressures are usually expressed in terms of head. Therefore, it can be said that cavitation in a pump is imminent when the net positive suction head (NPSH) ${ }^{*} \mathrm{H}_{\mathrm{s}}$ becomes equal to the dynamic for velocity) head $1 / 2 \rho u^{2}$.

In order to include cavitation effects in the list of dimensionless parameters for pumps, the additional parameter $S$ has been introduced by the definition

$$
S=\frac{N Q^{1 / 2}}{\left(g_{s}\right)^{3 / 4}}
$$

where $\mathrm{S}$ is the suction specific speed. The efficiency of a constant geometry pump can then be expressed (by considering cavitation but ignoring Re-effects) as $\eta=f(\varphi, S)$. It has become evident that pumps designed to resist cavitation have quite similar geometries. (73) Empirical test results have shown that (within a certain amount of scatter, of course) $S$ can reach about the value of 28 before a pump starts cavitating.

Considering again the case of liquid metal vs water, it can be seen that, in the term $H_{s}$, $S$ contains a specific fluid property (namely, the vapor pressure) only implicitly. Therefore, if the NPSH $\left(\mathrm{H}_{\mathrm{s}}\right)$ is kept the same for water and for liquid metal, cavitation tests conducted in water would be applicable to liquid metals (within the limits of this approximation).

It can be taken one step further: As the vapor pressures of sodium below $1200^{\circ} \mathrm{F}$ and of water around room temperature are normally both relatively small compared to $\mathrm{H}_{s}$, effects of different vapor pressures can be neglected altogether. However, as $\mathrm{P}_{\mathrm{v}}$ increases strongly with temperature, such an approximation would become less and less valid with increasing temperature. For example, it would make practically no difference whether the water temperature is $60^{\circ} \mathrm{F}$

*The net positive suction head (NPSH) is defined by $\mathrm{H}_{\mathrm{S}}=\mathrm{H}+\mathrm{H}_{\mathrm{a}}-\mathrm{H}_{\mathrm{v}}$, where $\mathrm{H}$ is the head of the liquid at the inlet of the pump, $\mathrm{H}_{\mathrm{a}}$ is the atmospheric head or that of the cover gas, $\mathrm{H}_{\mathrm{v}}$ is the vapor head corresponding to the temperature of the liquid. 
or $120^{\circ} \mathrm{F}$ for cavitation tests simulating $1200^{\circ} \mathrm{F}$ sodium. But if water at $190^{\circ} \mathrm{F}$ is used, then the vapor pressure of the water might become significant with respect to $\mathrm{H}_{\mathrm{s}}$. However, the deviation would be on the conservative side; i.e., if no cavitation is found in the water test, there would certainly be no cavitation in the corresponding liquid metal test either. It must be remembered, however, that the opposite statement (namely, if there is cavitation in the water there would also be cavitation in the liquid metal) is not necessarily true.

Unfortunately, pumps reach their peak efficiency just at the onset of cavitation; $(73,86,87)$ in trying to run a pump close to maximum efficiency, the danger of incurring cavitation is always quite close. Therefore, it might not often be possible to disregard the temperature dependence of vapor pressure as outlined in the preceding paragraph. ${ }^{(88)}$ In such cases the difference in $\mathrm{P}_{\mathrm{v}}$ for the corresponding conditions at different temperatures for water and for liquid metal must be added to the NPSH to determine the cavitation inception point. In tests conducted at Oak Ridge National Laboratory (ORNL) ${ }^{(83)}$ with $\mathrm{NaK}^{*}$ at $1500^{\circ} \mathrm{F}$, such a correction was used and the corrected data obtained from water tests agreed within about $5 \%$ with data obtained in corresponding $\mathrm{NaK}$ tests.

There are no apparent reasons to assume that cavitation damage will be similar in both water and liquid metals. Furthermore, cavitation details (which may be rather unimportant in many cases) such as delay, hysteresis, effects of surfaces, contamination, etc., are also likely to be completely different for water and for liquid metals. Therefore, to assess such damage effects, actual prototype testing in liquid metals must be done.

As cavitation usually occurs close to a body, the boundary layers are mostly affected. They could be drastically different with and without cavitation. However, as cavitation is to be avoided in liquid metal applications, this situation is of academic interest only, and will, therefore, not be discussed further.

Pumps for liquid metal use have apparently been designed, to date, according to conventional hydraulic principles. Water tests have usually been used to verify the design performance. In these tests, such parameters as pump head, mass flow, efficiency and cavitation inception were obtained. It was

* For the same temperature the vapor pressure of $\mathrm{NaK}$ is considerably higher than that of pure $\mathrm{Na} .(26)$

\section{LMEC $-68-4$}


found that the data from actual pump operation with sodium correlated fairly well with the water test data. In tests performed at ORNL, ${ }^{(89)}$ for example, pump head vs flow characteristics at several speeds were obtained first with water near room temperature and later with sodium at elevated temperatures. A slight difference between these two test fluids was observed, however. (Unfortunately, no quantitative data have been published on this discrepancy.) In view of the preceding discussions such a slight deviation is not surprising; in fact, it might even be expected. Grindell ${ }^{(89)}$ discussed an additional factor which might also contribute to this discrepancy: Because of the substantial differences in temperature (about $75^{\circ} \mathrm{F}$ for water vs $1200^{\circ} \mathrm{F}$ for sodium), the geometry of the pump might have changed somewhat; e.g., impeller clearances (which are as small as 0.020 in.) have quite an effect on pump performance and could change appreciably if impeller and casing were to expand at a slightly different and/or nonuniform rate.

Such expansion problems will become more severe as pumps get larger. Because these problems are rather unpredictable, it might be worthwhile to monitor the gaps in future liquid metal pump tests.

Because the elastic properties of the pump material depend on temperature, it is not possible to simulate in any water tests the stress levels that will occur in a pump operating in liquid sodium. Furthermore, the time-to-failure depends on temperature, as do the vibration characteristics. Any similarity rules that are applicable for the stress loading of geometrically similar pumps, ${ }^{(80,90)}$ therefore, are not applicable for water testing of liquid metal pumps.

Cavitation inception has also been correlated for pumps operating either in water or liquid metal. (83) This correlation was based on comparing an experimentally determined cavitation inception point to a predicted value which was obtained by adding to the water-test cavitation-inception point the difference in the vapor pressure between the hot liquid metal and the water.

Performance testing for liquid metal pumps has not been described in detail in the literature. It appears $(76,77,89,91)$ that in the United States pumps for liquid metal use have first been tested in water and have then been installed in the liquid sodium facilities for which they were intended. Practical experience with these pumps has generally been satisfactory. (78) This is probably due to the fact that their design was very much on the conservative side. With the 
exception of Reference 79 , there is practically no quantitative information available on how closely the results from the water tests were confirmed by actual operation in liquid metals. The same, incidentally, is true for bearings that have been used with sodium pumps in liquid-metal-cooled reactors. Also, only very little documented information on the actual development and design for these pumps and bearings can be found in the U.S. literature. It appears that standard hydraulic design procedures have been followed as if the pumps were only intended for use with water.

For an orderly development program for optimizing large sodium pumps, it would be imperative that actual hydraulic performance of the liquid metal flow inside the pumps be monitored by suitable instrumentation built into the pump. In this way theoretical predictions, water test data, and liquid metal test data can then be compared on a quantitative basis. Agreement with the pump manufacturers as to the type and the number of transducers required for this purpose should be reached at an early stage.

Other countries active in the LMFBR field have developed and tested liquid metal pumps also. In fact, it appears that some have done more extensive testing than the U.S. Russia has tested ${ }^{(92)}$ the pumps for its new BN350 LMFBR prototype at a $1: 4.5$ scale. Tests were then conducted in sodium with a dummy full-scale model for checking on the bearing design. A full-scale pump test facility (with 2-ft piping) was under construction during 1966 and is probably finished by now. As this facility comes closest to the AEC's planned SPTF, their operational test experience could be of value to the design and operation of SPTF.

Britain $(56,75)$ has also conducted extensive tests on the pumps for their Dounreay and PFR reactors. This work seems to be fairly well documented. They also used a special pump test rig (only l-ft pipe) for full-scale testing in liquid sodium. They tested their hydrostatic bearings (94) extensively first in water and then in paraffin.

France has developed its own pumps and bearings for its Rhapsodie and Phenix reactors. $(74,95)$ These modes: but systematic development and test programs have apparently been conducted by CEA on a long-range basis for at least 12 years. Extensive tests were performed with half-scale and full-scale pump and bearing models in water as well as in sodium. (The latest reports are not yet available here.) 
Germany and Japan have done very little liquid metal testing. Both countries are planning, however, to build test facilities for testing components in liquid sodium.

With the present surge of international interest in liquid metal testing procedures, it can be expected that during the next decade a testing technology will emerge which is far more sophisticated than any so far seen. In fact, the situation is closely analogous to the way in which the rapid development of wind-tunnel testing techniques was made possible during the thirties and forties. It appears that much could be gained from a mutual exchange of practical working experience with other test facilities, even if this would be done merely on a rather informal basis (e.g., patterned after the very successful international Supersonic Tunnel Association or the Jet Testing Association, etc.).

\section{SUMMARY ON PUMPS}

Testing the hydrodynamic performance of liquid metal pumps in water is well justified since such pumps are usually designed along well established hydraulic principles. Water testing is usually conservative and it can normally be expected that the pumps will actually perform hydraulically somewhat better in liquid metals. Cavitation testing is also possible in water. Generally, predictions of overall liquid metal pump performance based on water tests can be expected to be correct within about $10 \%$ or less. Only problems connected specifically with materials, high temperature, temperature transients, and vibrations should be investigated with the real fluid; i.e., hot liquid metals. Optimization testing for obtaining the best possible liquid metal performance should also be done with the real fluid. 


\section{MODEL TESTING OF BEARINGS}

The most important property of a lubricant in fluid film bearings is vis cosity (both dynamic viscosity $\mu$ and kinematic viscosity $\nu=\mu / \rho$ are important). Fortunately, the viscosity values of $\mu$ and $\nu$ for liquid metals are relatively close to those of water. Therefore, in testing bearings designed for liquid metal applications, water can be used with much greater confidence than can conventional lubricant oils which usually have much higher viscosities.

Dimensional analysis shows ${ }^{(2)^{*}}$ that the friction ${ }^{\dagger}$ coefficient $f$ of a journal bearing is a function of the geometry (e.g., $c / D, \ell / D$, etc.) and the dimensionless parameters $\mu \mathrm{N} / \mathrm{P}$ (if any moments on the bearing about an axis perpendicular to the shaft can be neglected); if $\mathrm{N}$ is the angular speed, $\mathrm{P}=\mathrm{W} / \ell \mathrm{D}$ is the bearing pressure, $\ell$ and $D$ are the length and the diameter of the bearing, and $c$ is the bearing clearance, then

$$
f=f_{b}\left(\frac{\mu N}{P}, \frac{c}{D}, \frac{\ell}{D}\right) .
$$

Dimensional analysis makes no predictions about the specific form of the function $f_{b}$. (As with pipe flow, it must be expected to be different whether the flow in the gap, clearance of the bearing, is laminar or turbulent.) Therefore, it would be very helpful for practical bearing design if a theory were available that would generate the proper functional relationship for $f_{b}$. Such a theory has been developed (first for laminar flow) from the original work of $O$. Reynolds and A. Sommerfeld. As one result of this theoretical treatment, a dimensionless parameter $S$ (Sommerfeld number) evolved, which is a combination of the simple parameter $\mu \mathrm{N} / \mathrm{P}$, previously mentioned, and the purely geometric ratio $\mathrm{R} / \mathrm{c}$ ( $\mathrm{R}$ = radius of the bearing):

*A more extensive treatment of dimensional analysis as it applies to bearing technology can be found in Reference 1. This book also contains probably the most comprehensive reference list on this subject ever assembled.

$\dagger f$ is defined as $f=2 T / W D$ where $T$ is the resisting torque of the bearing, $W$ is the load on the bearing and $D$ the diameter of the journal.

$\S I t$ is not feasible to outline this theory in detail here. There are several specialized texts $(96,97)$ available which show the development of these theories. 


$$
S=\frac{\mu N}{P}\left(\frac{R}{c}\right)^{2}
$$

Theory shows that

$$
S=\frac{\left(2+\epsilon^{2}\right)\left(1-\epsilon^{2}\right)^{1 / 2}}{12 \pi^{2} \epsilon},
$$

where $\epsilon$ is the eccentricity ratio of the bearing(96) The same theory has also provided a quantitative relationship for the friction coefficient $f$, at least for the laminar regime:

$$
f=f_{b}\left(S, \frac{c}{R}\right)=\frac{c}{R} \cdot \frac{1+2 \epsilon^{2}}{3 \epsilon}
$$

It can be seen that the friction coefficient is indeed a function of the geometry and the dimensionless parameters $\mu \mathrm{N} / \mathrm{P}$. ${ }^{*}$ Therefore, it is well justified by an established theory that liquid metal bearings can be tested for their fluid mechanical behavior by substituting water as the lubricant. In particular, the friction factor of a liquid metal bearing could be determined from water tests on the same bearing. It is also possible to match the $\mu$ of the water exactly to that of the liquid metal at its working temperature. In that case Sommerfeld numbers and friction coefficients are identical for liquid metals and for water within the framework of the assumptions on which this theory is based.

This convenient relationship breaks down as soon as the flow in the bea ring is no longer laminar. Unfortunately liquid metal bearings operate predominantly in this nonlaminar ${ }^{\dagger}$ regime, mainly because of the very low viscosity of the liquid metals.

*This is another instance in which intuitive dimensional analysis has been confirmed by strict mathematical procedures.

TQuite frequently this regime is termed superlaminar, which appears to be a poorly chosen term. Superlaminar flow encompasses not only the ordinary turbulent flow, but also a regime in between, which is characterized by a more or less regular pattern of Taylor vortexes. 
The criterion when laminar flow ceases to exist in a bearing is based on the fluid film $\mathrm{Re}$-number $\mathrm{Re}_{\mathrm{c}}=\mathrm{uc} / \nu$ (u being the circumferential velocity $\mathrm{u}=2 \pi \mathrm{RN}$, and $\mathrm{c}$ the median radial clearance of the bearing). When this number becomes larger than $41.3 \sqrt{\mathrm{R} / \mathrm{c}}$ the flow will become unstable and form regular Taylor vortexes. (36) When $\mathrm{Re}_{\mathrm{c}}$ exceeds about 1000 to 1500 the flow is said to become turbulent. (98)"

Here it becomes evident that for superlaminar flows the kinematic viscosity is the important fluid property to be simulated if it is desirable to compare liquid metal and water tests for bearings. (In the laminar regime, as discussed earlier, the dynamic viscosity $\mu$ is of primary importance.)

Occasionally a reduced Re-number is used ${ }^{(43)}$ in bearing applications. It again represents here, as it usually does, the ratio of the inertiaforces to the friction forces and is defined as ${ }^{\dagger}$

$$
\mathrm{Re}^{*} \cdot \frac{\mathrm{u} \ell}{\mathrm{v}}\left(\frac{\mathrm{c}}{l}\right)^{2}
$$

It s numerical value is much smaller than that of the regular Re-number; for a typical liquid sodium bearing of $20-\mathrm{cm}(8-\mathrm{in}$.) diameter, for example, and a $30-\mathrm{cm}$ length rotating at $600 \mathrm{rpm}$ with a clearance of $0.5 \mathrm{~mm}$, the reduced $\mathrm{Re}-$ number becomes $\overline{\mathrm{R}} \mathrm{e}^{*} \approx 7$, whereas the regular $\mathrm{Re}$-number is about 4000 .

The basic theories of lubrication are strictly valid only if $R e^{*}$ is smaller than one. Iterative approximations can be applied ${ }^{(43)}$ up to about $\mathrm{Re}^{*} \approx 5$ where the deviations reach about $10 \%$. It can be assumed, however, that typical liquid bearings reach $\mathrm{Re}^{*}$ numbers well above this value so that other more advanced theories have to be applied. During the last few years several attempts have been made to develop such theories which could be applicable for bearings in

*This criter $i o n$ apparently has been taken from pipe flow analysis, and it is therefore questionable whether it is justified here. Othe $r$ sources give much higher values. $(36,99,100)$ The whole point definitely requires further clarification, because it is not likely that the vortex structure disappears at a fixed

$\mathrm{Re}$-number and gives way to homologous turbulence.
$\dagger \mathrm{A}$ different definition of the modified $\mathrm{Re}$-number, $\bar{R} e^{*}=R^{2} \omega / \nu(c / R)^{2}$ is also used,(101) $(\omega=$ angular velocity). 
the superlaminar regime. (102-105) However, these rather complicated new theories have not yet been fully verified by experiments. "Furthermore, the validity of some of the underlying assumptions for these theories has even been found to be questionable. (109) Therefore, a successful development program for liquid metal bearings to be operated at superlaminar speeds (or more precisely at $\overline{\mathrm{R}} \mathrm{e}^{*}$ numbers $>1$ ) will be impeded somewhat by the present status of our limited theoretical knowledge. ${ }^{\dagger}$ As a consequence, the testing of newly designed $^{\S}$ and developed liquid metal bearings will assume a relatively high importance. The application of water tests for liquid metal bearing development purposes appears to be justified at least on an approximate basis. For instance, a test program aimed at an optimization of hydrodynamic performance of a particular bearing design could be done with water. References 113 and 114 list a whole series of nondimensional parameters that appear useful for such testing, and which have to a certain extent been justified by experimental verification.

As the viscosity of the lubricant plays such an important role in the behavior and performance of a bearing, it would be highly desirable if the viscosities of the model fluid could be closely matched with the viscosities of the prototype fluid. The following examples are intended to illustrate the importance of this point.

Liquid metal pump bearings could be operating in the turbulent regime at regular operating speeds, whereas the identical bearings tested with cold water could still be operating in the laminar regime at the same speed. As turbulent and laminar performance characteristics for bearings are completely different from one another, any model tests performed in cold water would, in such a case, be without any meaning for the hot liquid metal prototypes.

The mere simulation of Re-numbers for bearing testing is also not as straightforward as it is for the testing of pipe flows, for example, because the characteristic linear dimension in the Re-number is the clearance of the bearing.

*Additional theoretical work pertaining to the fluid mechanics aspects of bearing technology has recently been reported. $(99,105-108)$

tIt is important that efforts to add to the theoretical knowledge of liquid metal bearing behavior should also be encouraged. This kind of work should be pursued at various institutions, which might not be in complete agreement with each other.

§ractical information on hydrodynamic and hydrostatic bearing design can be found in References 105, 110-112,112a. 
Since the clearance itself is a highly critical factor affecting bearing perfor mance (e.g., it appears in the Sommerfeld number also), it cannot be changed around easily without possible major complications. For example, if water of $68^{\circ} \mathrm{F}$ were substituted in a model test for sodium of $1200^{\circ} \mathrm{F}$, it would be necessary to increase the clearance by a factor of 4 to obtain the same Re-number at the same same speed. In order to obtain the same Sommerfeld number, the clearance would have to be increased by a factor of $\sqrt{5}=2.25$. These two requirements are obviously incompatible.

If, by the same token, speed compensation would be applied to obtain the same Re-number, it would be necessary to increase the speed by a factor of 4 ; and in order to obtain the same Sommerfeld number it would be necessary to decrease the speed by a factor of 5. Again, it is not possible to meet both requirements at the same time. The basic reason for such diametrically opposing requirements is that in the case of the Sommerfeld number speed and viscosity form a product, whereas in the case of the Re-number they form a quotient.

Evidently by heating the water to higher temperatures (which normally also requires a pressurized system) it is possible to obtain a much closer matching of water and liquid metal viscosities (see page 14). However, it should be remembered that the heating and pressurization requirements complicate the test procedures considerably.

For such and similar reasons it would be preferable to use a test fluid such as acetaldehyde (see page 15) instead of water. Since both the dynamic and the kinematic viscosities of this organic liquid are equal at room temperature to those of liquid sodium at $1200^{\circ} \mathrm{F}$, both turbulence and inertia effects would be simulated in a quantitative way, which is impossible with water, even when it is heated to high temperatures. It also should be possible to apply model test results obtained with acetaldehyde directly to the corresponding liquid metal prototypes.

In cold water tests, a direct transformation of bearing model test results to liquid metal bearings is generally not possible. The water tests merely determine to what extent the theoretical procedure used to design the water bearing is being confirmed by the test data. If theagreement between theoretical 
predictions and experimental results is close (say within 10 to $20 \%$ ), there is valid reason to assume that the same design approach would work for liquid metals also.

As shown in the numerical example previously cited, the Re-number for a typical liquid metal bearing (to be used on the pump in a large LMFBR) is on the order of 4000 , which is in all likelihood in the turbulent regime. ${ }^{\dagger}$ Therefore, theoretical and experimental investigations of high speed bearings (of much smaller diameter) as conducted, for example, for the SNAP 50 program, can be of certain value for LMFBR projects. (It is the circumferential velocity $\omega R$, where $\omega$ is the angular velocity, which determines the flow phenomena.) The probability that the journal bearings to be used in LMFBR's will be vertically oriented, and thus will probably have relatively light loading, makes them similar to the SNAP 50 bearings, which were intended for use in space.

Hydrodynamic performance is certainly not the only characteristic which determines the overall quality of a particular bearing design. Other aspects such as rotor-bearing dynamics, cavitation and endurance behavior etc, are also highly important and are very difficult to analyze. Moreover there is a certain degree of interaction between the hydrodynamic and the latter characteristics, so that for a more thorough evaluation the system should be tested in its entirety. Quite generally the onset of a whirl or any other dynamic instability is very difficult to predict for a journal bearing (even in its laminar mode) because of the many factors upon which the whirl-impending frequency depends. Some of these factors are the rotor stiffness, the fluid film stiffness, the rotor polar moment of inertia, the rotor transverse moment of inertia, the external loads, etc. Because it is very difficult (if not impossible) to simulate all these conditions in a model it appears that a full-scale test of the original setup is the only sensible way of testing.

*Some of the latest theories ${ }^{(113)}$ appear to be good enough to expect such a close agreement.

$\dagger$ If such a pump is operated at reduced speed and/or if smaller clearances are used it might well be that the flow conditions could extend down into the laminar regime. Therefore laminar performance of liquid metal bearings is not without interest. 
For rotor dynamic tests $(111,115)$ there exists again the real possibility of using wate $r$ instead of liquid metals for the lubricant fluid as viscosities can, with some effort, be matched quite closely. (It should be remembered that matching of absolute and kinematic viscosities at the same time is impossible.) However, in such a case the operating temperatures would be quite different," which would cause material (e.g., elastic) properties to be different, and in turn might change the stiffness of the shaft, etc. Any structural distortion and misalignments caused by thermal gradients, which may adversely affect bearing performance, also cannot be simulated by water tests. Because it is difficult to determine if such effects are really significant in any particular case, the only sure way of obtaining reliable rotor dynamic test results is to test the bearing under actual operating conditions in the original setup.

A similar argument is used for cavitation effects. Although it is possible to test with water for the onset of cavitation, if the flow conditions are well defined, such conditions usually cannot be expected in actual operating bearings. For example, due to oscillations of the shaft non-stationary, localized underpressures, which could induce cavitation, may develop for short intervals. Furthermore, the different wettability characteristics of water and liquid metal as well as those of seizure resistance, wear, erosion, and corrosion (which are quite different for water and for liquid metals) would make any simulated water tests highly questionable.

Nevertheless, a few areas remain for which water testing is not only economically but technically justified; these are:

1) Tryout of new bearing concepts or designs to eliminate obvious failures. If a new bearing does not perform hydrodynamically in water it is useles to conduct more sophisticated tests in liquid metal.

2) If a new bearing type passes tryout testing successfully, more systematic development tests could be initiated in water with the objective of determing load-carrying capacity, and of optimizing the hydrodynamic performance.

*This is also true if tests were to be conducted with acetaldehyde. 
3) Many detailed aspects of the newly developed theories for superlaminar lubrication could be checked out in water tests.

If a new bearing has successfully passed through a series of development tests in water, the confidence that it also would work successfully. in liquid metals has increased very substantially. Therefore, water testing of bearings still retains some of its practical importance. It appears that it is the responsibility of the bearing manufacturer to design a bearing that is very likely to perform satisfactorily in liquid metals. Final confirmation of performance in a proof test performed with liquid metal, and possibly more comprehensive investigations that actually test the bearings as part of the whole system (which includes endurance tests under actual working conditions) could be performed on the test article. For such reasons an installation like SPTF should be outfitted with suitable instrumentation ${ }^{*}$ in order to monitor the bearing performance continuously with a sufficient degree of accuracy.

The arguments presented here for and against the various types of bearing tests are weighted differently for the many different kinds of bearings (hydrodynamic, hydrostatic, hybrid, bearings with tilted pads, with floating rings, etc.). In general, these discussions apply to all types but a more detailed investigation could isolate various differences.

Numerous liquid metal bearing development test programs have been performed during the last decade. (116-120) Most of them were aimed at developing high speed bearings for space applications. By far the most extensive tests were conducted at the Connecticut Advanced Nuclear Engineering Laboratory (CANEL) for the SNAP 50/SPUR pump bearing development program. Normally, water tests and liquid metal tests were performed on any type of bearing. According to Reference 121 the objectives of water and liquid metals testing were divided as follows:

\footnotetext{
*High temperature displacement transducers need to be developed for this purpose.

†This was an AEC sponsored program which was cancelled early in 1965 with less than half of its objectives accomplished. There are, however, many reports available that describe its initial test work in detail. These contain a wealth of potentially useful information which might prove quite helpful in starting any new liquid metal bearing test programs for LMFBR applications. It would require substantial background study in general bearing technology, however, to derive full benefit from it.
} 
New bearing designs are initially tested in a water environment at simulated conditions. Here actual performance data is readily available and many facets of the design can be evaluated. It is during water tests that the characteristics of the design are finalized. Based upon the results of these water tests the design can then be evaluated under appropriate conditions in a liquid metal endurance test.

Typical objectives of such liquid metal testing were: (1) to verify the load carrying capability of the bearing configuration in a gross sense; (2) to determine the compatibility of the bearing materials with the working fluid; and (3) to test the endurance of the selected bearing.

Bearings with diameters up to $4 \mathrm{in}$. were tested in this program in speed ranges of from 1000 to $12,000 \mathrm{rpm}$; putting these bearings in the same range of Re-numbers of interest for the LMFBR program.

R. Burton et al., at The Southwest Research Institute (SwRI) $(122,123)$ have been investigating some fluid mechanical characteristics (such as velocity and turbulence-intensity profiles) as they might occur in various types of bearings. They used an enlarged 6-ft diameter bearing model with air as the flow medium. The ultimate goal of these investigations was to aid in the development of liquidmetal-lubricated journal bearings. Air does not simulate liquid metal flow as closely as water but it appears to offer an advantage in that certain flow phenomena can be studied with conventional hot wire techniques.

About 6 years ago the performance of hydrodynamic journal bearings was investigated $(124,125)$ by using either water or liquid metal in the same bearing test rig. Although these tests were not primarily intended to compare the performance characteristics of water and liquid metal, it turned out that in some respects the liquid metal data differed considerably more from theoretical prediction than did the water data. No explanation was given. It could be that transducers were not as accurate with hot liquid metals as they were with water; but still these tests show that some caution is justified if water test data are applied to bearings designed for liquid metal applications.

Of all the bearing test programs performed during the last few years, the tests at CANEL $(119,120,126-132)^{*}$ and at Aerojet General, Scramento, (133)

*See page 46 for details. 
appear to be of the greatest value. In the latter case hydrostatic journal and thrust bearings for future huge rocket engine turbopumps (rocket thrust levels up to 6 million $\mathrm{lb}$, and pump power ${ }^{* *}$ up to $300,000 \mathrm{hp}$ ) have been investigated on a theoretical ${ }^{\dagger}$ and experimental basis. Water and (to a lesser extent) oil were used as test bearing lubricants. Highly improved theoretical relationships were also established both for laminar and for turbulent flow in the bearings. The water tests confirmed these theories for all major aspects of fully pressurized hydrostatic bearings.

According to the final report ${ }^{(133)}$ it is now feasible to reliably predict steady-state hydrostatic bearing performance with a low-viscosity lubricant ${ }^{\S}$ (which would include liquid metals). Computer programs were developed for this purpose. It appears that a liquid metal bearing could be designed with the help of such a computer program. It is possible then that development tests in wate $r$ would be unnecessary and all testing required for an actual verification of the theoretical design could be restricted to a proof test in liquid metal. However, tests would have to be $r$ un in the actual liquid metal environment for materials compatibility, endurance, cavitation, rotor-dynamic performance, etc. Therefore, a facility for testing liquid metal bearings under actual working conditions $^{* * *}$ (i.e., with liquid metals acting as lubricants) is still a necessity, whereas a water bearing test facility no longer appears to be a real requirement.

*Compared to a pump power level of only $6000 \mathrm{hp}$ for SPTF. Although these pumps are of a much higher power level than the pumps for future LMFBR's, their geometrical size appears to be comparable. For example, the rocket pumps have a shaft diameter up to $7.4 \mathrm{in.}$, whereas the latest Byron Jackson estimates call for 8.7 in.; the bearing loads are about $28,000 \mathrm{lb}$ for the rocket pump vs an estimated $14,000 \mathrm{lb}$ for the liquid metal pump. The main difference between the pumps seems to be the rotational speed (roughly up to $20,000 \mathrm{rpm}$ for the rocket pump vs up to $1000 \mathrm{rpm}$ for the liquid metal pump) and the developed head (roughly up to 4000 psi vs $200 \mathrm{psi}$ ). The actual Aerojet water tests for the journal bearings were done with a journal diameter of 4.5 in. No rotor dynamic tests have been performed. Bearing power loss (order of $100 \mathrm{hp}$ ) and lubricant flow (order of $100 \mathrm{gpm}$ ) were rather low.

TIt is worthwhile to note here that their theories are based on fundamental work done by Tao.(104)

$\S A$ recently completed independent project(113) essentially confirms such claims. Overall predictions, which were based on turbulent lubrication theory (including inertia effects), were substantiated in actual performance tests within about 10 to $15 \%$. The lubricant in this case was a cryogenic liquid.

*:* The planned SPTF at LMEC could be used if it would be instrumented for this purpose. 
Quantitative test data on journal bearing performance, using a hot liquid metal as the lubricant, are still scarce. "There are no known references in the literature to a direct quantitative comparison of hydrodynamic test data obtained from the same bearings operating in water and in liquid metals, respectively. It can be concluded through an indirect comparison that the data will agree within approximately 0 to $50 \%$, depending on test conditions and the source of information. (Such an indirect comparison can be made by comparing water test data ${ }^{\dagger}$ and liquid metal test data to the same theoretical predictions.) No systematic trends can be detected for the deviations. Generally the water tests show closer agreement with theory. Deviations from theory are usually in the same direction in both cases, which could mean that the test data for water and liquid metals are more often in closer agreement with each other than with theoretical predictions.

The current activity in hydrodynamic and hydrostatic bearing research in the USA seems to be at a rather low level. Most of the recent bearing programs (References 113,122, 133, 138, and 139) sponsored by either NASA, USAF, or AEC have been cancelled or curtailed.

\section{SUMMARY AND RECOMMENDATIONS ON BEARINGS}

According to the latest literature on journal bearings, theories used to predict the hydrodynamic performance $\S$ of various types of such bearings (socalled hydrodynamic, hydrostatic, hybrid bearings, etc.) are advanced enough to allow the actual design of a bearing for a set of given requirements with a reasonable degree of confidence. This is true for bearings operating in the laminar as well as in the superlaminar regime with either water or liquid metals as lubricants. Though to a much lower degree of confidence, these theories can also be used to predict the rotor-dynamic performance $* *$ of such bearings.

*Further experimental work of recent origin has been reported in References 134 to 137 .

†In some cases $(116,133)$ the deviations in water test data have been as small as 0 to $10 \%$.

§Hydrodynamic performance includes such items as torque, load carrying capacity, power loss, mass flow, etc.

**Rotor-dynamic performance includes such items as critical speeds, onset of instabilities, imbalance response, etc. 
Water tests appear to have been primarily helpful in verifying and/or optimizing an unresolved design; however, the need for such tests has decreased somewhat over recent years. Furthermore, as only the fluid mechanical characteristics of a liquid metal bearing can be tested in water with good confidence, the many other characteristics (such as rotor dynamics, endurance, etc.) still must be tested in their real environment; i.e., in liquid metals at their actual working temperature. Such liquid metal tests would: (1) confirm (proof test) the hydrodynamic (fluid mechanics) performance as predicted on the basis of the theoretical design and/or water tests; (2) provide data on such uncertainties as materials compatibility, cavitation, erosion and corrosion, endurance, etc.; and (3) generate complete rotor dynamic performance data, which can be obtained reliably only in a real environment.

The necessity for testing liquid metal pump bearings in their working environment also implies that they should be tested as integral parts of an actual pump being operated throughout its entire operating range. It appears that this kind of testing $c$ an be done best in a combined liquid metal pump and bearing test facility; the advantage being that both bearing and pump testing could be accomplished simultaneously in the same facility. It also would automatically account for any possible interaction between the hydrodynamic and rotordynamic variables of the bearing system. 


\section{MODEL TESTING OF FLUID SEALS}

Of the various types of seals used or proposed for liquid metal applications, only the viscoseal (140-146) and the centrifugal dynamic seal (147) are of interest here because they are the only types that rely on fluid mechanic principles.

Since both theoretical knowledge and practical experience with these seals are still rather limited, testing assumes a special significance.

As long as the flow in the seal is laminar, the dimensionless parameter $\Lambda$ characterizes the performance of the viscoseal:

$$
\Lambda=\frac{\mu \omega \mathrm{d} \ell}{\Delta \mathrm{Pc}^{2}} \text { (sealing coefficient) }
$$

where

$$
\begin{aligned}
\mu & =\text { the absolute viscosity of the sealing liquid } \\
\omega & =\text { the shaft rotational speed } \\
\mathrm{d} & =\text { seal diameter } \\
\ell & =\text { seal length } \\
\mathrm{C} & =\text { clearance } \\
\Delta \mathrm{P} & =\text { pressure differential across the seal. }
\end{aligned}
$$

The only material constant in that parameter is $\mu$ and therefore liquid metals can be substituted by water if their viscosities are matched.

In the turbulent regime (which is less understood) the Re-number

$$
\operatorname{Re}=\frac{\omega \mathrm{dc} \rho}{\mu}
$$

becomes the important parameter which determines the sealing coefficient. In this case the kinematic viscosities should be matched if liquid metals are to be simulated in water experiments.

Although viscoseal tests have been performed in water and in liquid metals, $(142,150)$ there are still a number of little understood areas (such as gas ingestion, etc.) which cannot be reliably simulated by a different test fluid. It is, therefore, still necessary to proof test any liquid sodium seal with the hot liquid metal even if its performance had been satisfactory in water tests.

$$
\text { LMEC-68-4 }
$$




\section{LIQUID METAL HEAT TRANSFER PROBLEMS AND MODEL TESTING}

\section{A. FORCED CONVECTION HEAT TRANSFER}

Whereas the viscosities of water and sodium are of the same order of magnitude (see page 13), their thermal properties are vastly different. The thermal conductivity for sodium is about 150 times that of water ( $\lambda \approx 0.5 \mathrm{kcal} / \mathrm{mhr}{ }^{\circ} \mathrm{C}$ for water at $20^{\circ} \mathrm{C}$ vs $\lambda \simeq 74 \mathrm{kcal} / \mathrm{mhr}^{\circ} \mathrm{C}$ for sodium at $100^{\circ} \mathrm{C}$ ); the thermal diffusivit $y^{\prime}$ of sodium is about 480 times that of water $\left(\mathrm{a}=0.05 \times 10^{-2} \mathrm{~m}^{2} / \mathrm{hr}\right.$ for water at $20^{\circ} \mathrm{C}$ vs $\mathrm{a}=24 \times 10^{-2} \mathrm{~m}^{2} / \mathrm{hr}$ for sodium at $100^{\circ} \mathrm{C}$ ); the specific heat of sodium is about 3 times smaller than that of water $\left(c_{p}=1 \mathrm{kcal} / \mathrm{kg}{ }^{\circ} \mathrm{C}\right.$ for water at $20^{\circ} \mathrm{C}$ vs $c_{p}=0.33 \mathrm{kcal} / \mathrm{kg}^{\circ} \mathrm{C}$ for sodium at $100^{\circ} \mathrm{C}$ ). As a consequence, the Prandt] number, which is one of the important nondimensional parameters for heat transfer, is about 600 times larger for sodium than for water ( $\operatorname{Pr} \approx 7$ for water at $20^{\circ} \mathrm{C}$ vs 0.0115 for sodium at $100^{\circ} \mathrm{C}$ ).

These large differences by themselves do not necessarily prohibit a water simulation of liquid metal heat transfer. However, to arrive at any meaningful conclusions, dimensional analysis must be used. Fortunately, dimensional analysis has been particularly successful in the field of heat transfer. Besides the already introduced dimensionless parameters such as Re-number and pressure coefficient,

$$
\mathrm{Re}=\frac{\mathrm{Lu}}{v}, \mathrm{Eu}=\frac{\Delta \mathrm{p}}{\frac{1}{2} \rho \mathrm{u}^{2}},
$$

a whole series of additional parameters can be formed. The more important ones are

1) Nusselt number: $\mathrm{Nu}=\mathrm{hL} / \lambda$ ( $\mathrm{L}$ is a characteristic length as in the Re-number, $\lambda$ is the heat conduction coefficient, and $h$ is a heat transfer coefficient defined as $h=q / \Delta T$, with $q$ being the heat flux density generated by the temperature difference $\Delta T$ );

2) Prandtl number: $\operatorname{Pr}=\nu / a$ (which is the ratio of the kinematic viscosity to the thermal diffusivity, and as such is a pure material constant which, of course, is temperature dependent);

\section{LMEC-68-4}


3) Peclet number: $\mathrm{Pe}=\mathrm{Re} \cdot \mathrm{Pr}$; and

4) Stanton number: St $=\mathrm{Nu} / \mathrm{Pr}$ are frequently used, convenient combinations of the more fundamental parameters above, although they are not really necessary for the treatment of heat transfer.

In cases where effects of the entrance region of a pipe becomes important, a dimensionless parameter, the Graetz-number $\mathrm{Gz}=\operatorname{Re} \cdot \mathrm{Pr} \cdot \mathrm{D} / \mathrm{X}$ is occasionally used, $(151,152) \mathrm{D}$ being the pipe diameter and $X$ the distance from the entrance.

For convective heat transfer to or from a wall, dimensional analysis yields the relation ${ }^{(2)} \mathrm{Nu}=\mathrm{f}(\mathrm{Re}, \mathrm{Pr})$. As usual, it does not specify the particular form of the relationship. This must be derived either on a theoretical basis from the underlying differential equations or from experiments. "The basic differential equations (Navier-Stokes equation, equation of continuity, and equation of heat conduction) can be written in a dimensionless form which contains the Re-number, the $\mathrm{Pr}$-number, and the Nu-number as coefficients, which confirms that the solution must be of the form previously cited. (This is strictly true only for convective heat transfer processes in which viscous dissipation and compressibility are negligible, as it is for water and for liquid metals). The exact solution of this system of differential equations depends on boundary conditions and is not generally obtainable because of the mathematical difficulties involved. Only in certain special cases has an approximate solution been found. In particular, the important case of heat transfer in tubes has been investigated extensively.

It has been shown theoretically ${ }^{(36)}$ that for laminar flow in tubes the relation is of an even simpler form: $\mathrm{Nu}=\mathrm{f}(\mathrm{Pe})$; this is a special case of $\mathrm{Nu}=\mathrm{f}(\mathrm{Re}, \mathrm{Pr})$. In general, experiments ${ }^{(12)}$ have shown that the relation is approximated by $\mathrm{Nu}=0.025 \mathrm{Re}^{0.8} \mathrm{Pr}^{0.4} .^{\dagger}$ Where $\operatorname{Pr} \approx 1$ (e.g., for gases) the approximation is simply $\mathrm{Nu}=0.025 \mathrm{Re}^{0.8}$. If a logarithmic velocity profile is assumed for the turbulent flow $(\operatorname{Pr} \approx 1)$, a similar equation can be derived on purely theoretical grounds: ${ }^{(36) \S} \mathrm{Nu}=0.04 \mathrm{Re}^{0.75}$.

*In many instances of turbulent heat transfer it is derived partly from theoretical considerations and partly from empirical data.

$\dagger$ Also known as Dittus and Boelter equation. \$Such basic relations have already been derived in various forms by Reynolds, Nusselt, and Prandtl in the time before 1910. Numerous attempts have been made since, and are still being made, to improve on these simple relations. 
With their high thermal conductivities (or $\operatorname{Pr} \ll 1$ ), the liquid metals are in a category by themselves and therefore the preceding relations do not generally apply to liquid metals." Under turbulent flow conditions the eddy conduction and the molecular conduction of heat interact throughout the turbulent portion of the flow. (155) be neglected. Therefore, the heat transfer equations for liquid metals have to be augmented by terms that stand for the molecular heat transfer contribution. This can be done in a fir st approximation by a simple constant: $\mathrm{Nu}=$ const $+\mathrm{f}(\operatorname{Re}, \operatorname{Pr})$. It can be shown theoretically that the upper limit for this constant is about $8 .(156)$ Many versions of this equation based on empirical results have been proposed over the recent years. One that has been proposed by Lion ${ }^{(157) \S}$ has been cited most frequently: $\mathrm{Nu}=7+0.025 \mathrm{Pr}^{0.8}$.

It is rather difficult to judge which of the various versions is the best, as liquid metal heat transfer data usually show a relatively high degree of scatter. The version favored presently ${ }^{(159)}$ seems to be $\mathrm{Nu}=5+0.025 \mathrm{Pe}^{0.8}$, as proposed by Subbotin. (160) To the uninitiated a formila of this type might suggest that there is a possibility of modeling turbulent heat transfer phenomena of liquid metals in water. There are, however, the following obstacles:

1) The constant 5 (or whatever it may exactly be) ${ }^{*}$ is not present in the corresponding equation for water (i.e., it is practically zero in that case).

*This is why heat transfer in regular media (except liquid metals) is easier to treat and is therefore more advanced than liquid metal heat transfer.(153) For the same reasons most general texts on heat transfer are of little value in solving liquid metal problems. A new book by Kays (154) tries to give the liquid metal heat transfer problems some consideration.

$\dagger$ In laminar flow, where molecular conduction controls the heat transfer process, there is no fundamental difference between the various fluids; therefore, the nondimentional formula can be applied. In this case, water can be used to simulate heat transfer in liquid metals. Unfortunately, most liquid metal flow applications occur in the turbulent regime. Therefore, this laminar case is of very limited interest only.

§It does not come as a surprise that Lion's empirical equation has this particular form if it is remembered that it is valid for liquid metals only, and then only approximately. Other equations, such as $\mathrm{Nu}=0.625 \mathrm{Pe}^{0.4}$, have also been proposed. $(158)$ They are said to be more consistent with later liquid metal experimental data. These two equations are quite different indeed, and an estimate can be made as to the quality of the experimental data if they can be fitted by both equations.

**Many of the apparent discrepancies seem to be due to incorrect measurements. Recently it was shown by very careful experiments(151) that the theoretical value of 4.36 is actually correct. 
2) The use of the Pe-number in this formula may be misleading. It must be remembered that the Pe-number consists of the product of the Re-number and the Pr-number, which in the original theoretical derivation carry different exponents. It is only for correlations within material groups of similar Pr-numbers (such as liquid metals) that the use of Pe is justified.

For modeling liquid metal heat transfer in water, therefore, the Re-number and $\mathrm{Pr}$-number must be the same in the prototype and the model case. This is impossible, however, as the $\operatorname{Pr}$-number is a pure material constant which is (as shown in the preceeding text) completely different for water and for liquid sodium. As long as Lion's or Subbotin's equations are used exclusively for liquid metals the simpler version using the $\mathrm{Pe}-$ number is justified as an approximation. * But even assuming a Pe-number simulation were possible for heat transfer, Re-number simulation would be necessary also for the fluid flow itself. However, this leads to a requirement which is highly impractical for the follow ing reason: As the $\mathrm{Pr}$-number is about 600 times larger for water than it is for liquid sodium, the Re-numbers in a water test must be 600 times smaller than those in the prototype liquid metal case, if it is required that Peclet numbers are equal in both cases. For example, if the Re-numbers for a typical flow in a reactor core is 60,000 then the Re-number for a corresponding wate $r$ model test would have to be 100 . The latter is clearly in the laminar regime, whereas the former is in the turbulent regime. For proper modeling both cases must be in the turbulent regime, which implies that the Re-number for the core flow would have to be on the order of about 2 million or higher. This, however, is impractical.

Thus, it can be concluded that liquid metal heat transfer under turbulent flow conditions cannot be simulated by water experiments. The question whether pure scale modeling and modeling at different temperature is possible for liquid metal heat transfer is answered in the following text.

* Because liquid metals do not have truly identical Pr-numbers (they are all small, but still they are different), and as $\operatorname{Pr}$-numbers depend on temperature, these equations give approximate results only. This is borne out by experimental data(159) and more recent theories. (161) However, for medium and high $\mathrm{Re}$-numbers $(\operatorname{Re}>3000)$ these differences become almost negligible, according to the same theory. 
As it is especially difficult to test liquid metals at elevated temperatures (such as the 800 to $1200^{\circ} \mathrm{F}$ range), there is a question as to whether testing with liquid metals (e.g., NaK) at room temperature will give useful results.

If the small differences in the Pr-number among various liquid metals at different temperatures are ignored the results from $\mathrm{NaK}$ model testing would obey the general formula

$$
\mathrm{Nu}=5+0.025 \mathrm{Pe}^{0.8}=5+0.025(\operatorname{PrRe})^{0.8}
$$

The $\operatorname{Pr}$-number for sodium at $1200^{\circ} \mathrm{F}$ is 0.004 , compared to 0.02 for $\mathrm{NaK}$ at $200^{\circ} \mathrm{F},{ }^{(26)}$ which means they are different by a factor of 5 . Nusselt numbers will therefore differ by a factor of almost 4 for large $R e-n u m b e r s(R e \rightarrow \infty)$ and will not differ at all for small $\mathrm{Re}$-numbers $(\mathrm{Re} \rightarrow 0)$.

This is a rather poor method for comparison; therefore, it is recommended that Pe-numbers be matched as far as possible. This would yield more acurate data on heat transfer, although the fluid flow data (such as pressure drop, etc.) may be different. As the Pe-number $\mathrm{Pe}=D u \rho c_{p} / k=D u / a$ carries both material and fluid flow variables, any difference in the the rmal diffusivity $a=k / \rho c_{p}$ of the two different liquid metals ${ }^{*}$ can be compensated for by a change in velocity $u$, for example.

Model testing with liquid metals for the investigation of heat transfer in liquid-metal-cooled reactor cores has been reported in the literature. $(162,163)$ In such a case it appears to be necessary to use a full-scale or even a scaledup model of a subassembly or part of a subassembly.

As briefly mentioned before, it is no longer apparent that for model testing in liquid metals (as in using $\mathrm{NaK}$ at room temperature to predict heat transfer in liquid sodium at $1200^{\circ} \mathrm{F}$ ) the familiar and useful concept of hydraulic diameter ${ }^{(58)}$ can be used with confidence because of the greater importance of molecular conduction of heat in turbulently flowing liquid metals. By using this concept for non-metallic fluids it is possible to use basic heat transfer data for

*Roughly a factor of 2 between $1200^{\circ} \mathrm{F} \mathrm{Na}$ and $200^{\circ} \mathrm{F} \mathrm{NaK}$.

LMEC -68-4 
water flow through circular tubes to predict $\mathrm{Nu}$-numbers for water flow parallel to a bundle of rods by evaluating the hydraulic diameter for the latter. Such methods are found to be invalid for liquid metal systems. (164)

More elaborate theories of heat transfer in general, and liquid metal heat transfer in particular, have been proposed. (161,165-168) None of them, however, has been completely successful; they still rely to a certain extent on empirical data. The stumbling block is very basically the inability to deduce theoretically the distribution of flow parameters in turbulent boundary layers $(169)^{*}$ (including also the flow in ducts). For example, the so-called law of the wall has not been derived yet on purely theoretical assumptions, although efforts are continuously being made $e^{(174,175)}$ to accomplish this.

For heat transfer in particular, the distribution of eddy viscosity and eddy diffusivity, which are functions of Re and Pr numbers, are urgently needed. $(154,155)$ As the potential usefulness of successful model testing depends to a large degree on the status of the underlying theories, any progress in those theories would indirectly also help the experimentalist in his efforts to obtain meaningful test data.

As heat transfer (or more precisely, temperature) affects the transport properties of the fluid heat transfer medium, certain indirect effects on the mechanics of the fluid flow must be expected, which in turn can affect the heat transfer. For ordinary fluids like water (especially when close to their critical temperature) this interaction can be significant, $(176,177)$ whereas for liquid metals it is usually quite small and the refore negligible. (178-180)

The influence of heat transfer on the velocity profile of liquid metal flow has been investigated experimentally ${ }^{(181)}$ and it was found that for heat transfer rates up to about $30,000 \mathrm{kcal} / \mathrm{m}^{2} \mathrm{hr}{ }^{\circ} \mathrm{C}$ there was no deviation from the isothermal

*The topic of turbulence(170-173) has certainly been the most challenging and stubborn problem of fluid mechanics in this century. Its final solution would automatically solve a host of other problems in many different fields of engineering science. 
case.* Within the accuracy of the experiments $( \pm 15 \%)$ it was also shown that the basic relationship of $\mathrm{Nu}=5+0.025 \mathrm{Pe}^{0.8}$ is valid at least up to these values of heat flux densities. Thus it is possible (within the limits cited) to treat the fluid dynamic and the heat transfer aspects of liquid metal flows separately. Accordingly it is justified to determine for example, the pressure drop through a liquid metal heat exchanger by performing a corresponding model flow test in water. (However, it is not possible to derive any quantitative liquid metal heat transfer data from such water tests.) It has been found, though, that relatively large (up to about 50\%) discrepancies between experimental water data and theoretical predictions (for water) can exist. (177) No plausible explanation has been given for this strange behavior for liquid metals. The typical large spread of experimental heat transfer data $(182)$ is well known, although it seems to have narrowed down in recent years ${ }^{(159)}$ due to more refined measuring techniques. But reliable heat transfer data are still very hard to obtain, particularly for liquid metals at high temperatures.

Some of the causes for data discrepancies in heat transfer model testing can certainly be found in deviations from the conditions for which the basic theories and model laws have been derived. ${ }^{\dagger}$ For example, the effects of wall roughness, contaminations in the fluid, effects of short entrance lengths and channel shape, bends, elbows, restrictions in the ducts, boundary layer transition and separation, variable thermal properties, heat flow along flow axis, buoyancy, etc., all contribute to such deviations in varying degrees. It can be restated that, apart from purely geometrical ratios, the influence of these various effects depends on dimensionless parameters such as $R$ e-numbers and Pr-number. Generally, for heat transfer, they tend to be different for liquid metals (small Pr-numbers) than for water (large Pr-numbers).

*Because heat transfer rates for sodium on stainless steel interfaces can go as high as $1.5 \times 10^{6} \mathrm{kcal} / \mathrm{m}^{2} \mathrm{hr}{ }^{\circ} \mathrm{C}(5 \mathrm{l})$ there is the distinct possibility that at these much higher rates the fluid dynamic characteristics of the flow will be affected. Indeed such an effect must eventually be expected if only the heat transfer rates could be increased far enough, before the onset of boiling. Therefore, experiments with increasingly high heat transfer rates might be needed for the development and safe operation of high flux LMFBR's.

†These causes of data discrepancies include those already discussed concerning flow in ducts (see page $18 \mathrm{ff}$ ). 


\section{B. HEAT TRANSFER BY NATURAL CONVECTION}

Heat transfer by natural convection may play a role in removing decay heat in a nuclear reactor; therefore it is discussed here briefly.

Dimensional analysis predicts that the free convection heat transfer, characterized in dimensionless form by the $\mathrm{Nu}$-number $\mathrm{Nu}=\mathrm{hL} / \lambda$ depends on the geometry, the $\mathrm{Pr}$-number and a new parameter $\mathrm{Gr}=\mathrm{g} \beta \mathrm{L}^{3} \Delta \mathrm{T} / \nu^{2}$ (Grashof number), where $\beta$ is the coefficient of thermal expansion and $\Delta \mathrm{T}$ is a characteristic temperature difference. Differential similitude ${ }^{(12)}$ has verified these predictions (provided viscous dissipation effects can be neglected, which is usually the case for liquid metals). Therefore, the similarity law for geometrically similar cases can be stated in the form:

$$
\mathrm{Nu}=f(\mathrm{Gr}, \mathrm{Pr})
$$

Compared to forced convection, the Re-number has merely been replaced by the Gr-number. (For a physical interpretation of Gr it can be shown that $\mathrm{Gr}$ is proportional to $\mathrm{Re}^{2}$ of the convection layer. Grashof numbers can become quite large, up to $10^{12}$ or more). As a rough approximation for laminar free convection the relationship can be written in the following form:

$$
\mathrm{Nu}=f(\mathrm{Gr} \times \mathrm{Pr})=f(\mathrm{Ra})
$$

where $\mathrm{Ra}=\mathrm{Gr} \times \operatorname{Pr}$ is known as the Raleigh number, which is again analogous to the laminar forced convection case, where $\mathrm{Nu}$ is a sole function of the $\mathrm{Pe}-$ number. A somewhat different approximation has been proposed for liquid metals in the form

$$
N u=f\left(G r \times \operatorname{Pr}^{2}\right),
$$

which again shows that liquid metals behave differently from nonmetallic liquids such as water. Specifically, in the range of $\operatorname{GrPr}^{2}$ from 10 to $10^{4}$

$$
\mathrm{Nu} \approx 0.53 \sqrt{\mathrm{Gr}} \times \overline{\operatorname{Pr}^{2}}
$$

\section{LMEC - $68-4$}


In order to obtain more exact relationships, the differential equations for laminar free convection have been solved for a few very simple cases. ${ }^{(183)}$ In the case of convection on a vertical isothermal plate for laminar boundary layer flow conditions the Nusselt number relation becomes

$$
\mathrm{Nu}=\mathrm{F}(\mathrm{Pr}) \sqrt[4]{\mathrm{Gr}}
$$

The function $F(\mathrm{Pr})$ is not available in closed form and must be determined from tables or graphs. It changes considerably with Pr; for example, its numerical value for liquid metals $(\mathrm{Pr} \approx 0.01)$ is 0.076 whereas for water it is approximately 1. For other geometrical configurations the function $F$ is different again. In many cases it may be practically impossible to determine the $F(P r)$ function because of mathematical difficulties. Generally it can be said that computations involving liquid metals $(\operatorname{Pr} \ll 1)$ are more complicated and time consuming than those for medium and high Pr-numbers (such as for water).

Because of the strong and often unknown Pr-number dependence, model tests for simulating liquid metal free convection in water are generally not possible. But even in those very simple cases where the Pr-number dependence could be accounted for analytically, it would be necessary to match the Grnumbers if such water testing would be considered. The Gr-number contains two material constants ( $\nu$ and $\beta$ ); both are approximately of the same order of magnitude for water and for liquid sodium. But $\mathrm{Gr}$ also contains a characteris tic temperature differential $\Delta T=T_{0}-T_{\infty}$ which, in the case of the vertical plate, is the difference between the plate temperature $T_{0}$ and the free stream temperature $\mathrm{T}_{\infty}$ at infinity. The temperature profile of the thermal boundary layer is completely different ${ }^{*}$ for water and for liquid metals ${ }^{(154)}$ and they cannot generally be matched by affine transformation in a finite distance. Therefore, in most practical cases (e.g., convection in a vertical duct) it is virtually impossible to match Gr-numbers for geometrically similar cases in water and in liquid metal.

*If it were known quantitatively in what way they are different it might be possible to compensate for a smaller $\Delta \mathrm{T}$ in liquid metals by using smaller dimensions $\left(L^{3}\right)$ in water. 
If an attempt is made to model forced convection heat transfer in a geometically similar but smaller model, using the same heat transfer medium (which implies having equal Pr-numbers), similarity analysis would require that the Re-number be equal. If, however, free convection contributes to the heat transfer in either one or in both cases, the accuracy of resulting data would be adversely affected because it is practically impossible to simulate Re and Gr simultaneously. It can be shown ${ }^{(12)}$ that free and forced convection are of the same magnitude if the Gr-number is of the order of $\mathrm{Re}^{2}$; in fact, the ratio Gr/Re $e^{2}$ can be considered as an indication of the relative effect of buoyancy upon forced convection.

This problem possibly could explain some observed discrepancies in connection with model testing for liquid metal heat transfer. For example, if Nu-numbers were determined in a small model at greatly reduced scale, the velocities of the liquid metal flows in these models were in all likelihood so high that there was practically no contribution due to free convection heat transfer, while in the full-scale model there could very well have been such a contribution. In a situation like this the model data (pressuredropand heat transfer) would generally correlate fairly well with theoretical predictions, which would make them appear even more reliable, whereas only the full-scale data would show all the discrepancies.

Only laminar free convection $(184)$ has been considered to this point. If Gr-numbers become large enough (approximately $>10^{8}$ ), transition to turbulence will occur. No well developed theory exists for this region because of the highly complicated nature of the flow. $(167,168,185)$ Consequently, there are no reliable similarity laws either. There are, of course, a number of empirical and semi-empirical correlations, but they are of limited value. It must again be concluded, therefore, that water model testing does not yield any reliable results for turbulent free convection in liquid metals. The model testing with liquid metals (as briefly outlined on page 57) apparently has not been tried yet under these conditions, but it appears likely that it is possible.

\section{TWO-PHASE F LOW AND HEAT TRANSFER}

Because the boiling mechanism is highly complicated, a pure analytic expression for the two-phase heat transfer, derived from basic theoretical 
relations, has not yet been obtained. The same can be said even of the basic hydrodynamics of two-phase flow itself, which means that such things as pressure drops or velocity profiles (including void fraction, etc.) cannot be predicted analytically. It is not surprising, therefore, that dimensional analysis is employed frequently to facilitate finding empirical correlations for two-phase flow and heat transfer data. There are quite a number of special dimensionless parameters that apply specifically to two-phase flow; however, they will not be discussed here. $(186,187)$ Withrespect to heat transfer correlations, Rohsenow (188) and Forster-Zuber ${ }^{(189)}$ have shown that the familiar general type of the correlation $\mathrm{Nu}=\mathrm{f}(\mathrm{Re}, \mathrm{Pr})$ is also valid for two-phase heat transfer, provided that the dimensionless parameters are suitably defined. In the latter case, the $\mathrm{Nu}$-number $\mathrm{Nu}_{\mathrm{b}}$ is defined as the ratio of the boiling heat transfer $\mathrm{r}$ ate to the conduction heat transfer rate through the liquid film:

$$
N u_{b}=\frac{\delta q^{\prime \prime}}{k_{l}\left(T_{w}-T_{b}\right)},
$$

where

$$
\begin{aligned}
& \delta=\text { thickness of the liquid film } \\
& \mathrm{k}_{\ell}=\text { thermal conductivity of the liquid } \\
& \mathrm{q}^{\prime \prime}=\text { heat flux } \\
& \mathrm{T}_{\mathrm{w}}=\text { wall temperature } \\
& \mathrm{T}_{\mathrm{b}}=\text { vapor temperature } \\
& \text { subscript } \mathrm{b}=\text { bubbles. }
\end{aligned}
$$

The $\mathrm{Re}$-number $\mathrm{Re}_{\mathrm{b}}$ is defined here as the ratio of the bubble inertial force to the liquid viscous force and indicates, therefore, the intensity of liquid agitation induced by the bubble motion:

$$
\operatorname{Re}_{b}=\frac{\rho_{b} V_{b} D_{b}}{\mu_{l}}
$$


The $\operatorname{Pr}-n u m b e r \operatorname{Pr}_{\ell}$ of the liquid is defined as the ratio of the kinematic viscosity $(\nu)$ to the the rmal diffusivity $(a)$ of the liquid: $\operatorname{Pr}_{\ell}=\nu / a$.

In specific terms the Forster-Zuber correlation can be written as $\mathrm{Nu}=$ $0.0015 \mathrm{Re}_{\mathrm{b}}{ }^{0.62} \times \mathrm{Pr}_{\ell}{ }^{0.33}$. Although this relation was primarily intended for boiling water heat transfer, it was also found to be applicable (within a certain error margin) to boiling liquid metal heat transfer; ${ }^{(190)}$ several similar relations have also been found applicable. (191) Because of the widely different temperature profiles of boiling liquid metals and water, and the resulting difference in boiling behavior this finding comes somewhat as a surprise.

Correlations for the pressure drop in two-phase flow have been developed by Martinelli et al. (192) If the pressure drop $\Delta p$ over a length of pipe $\Delta l$ is signified in one-phase gas flow by the subscript $g$ and in two-phase flow by the subscript $\ell$ the correlation can be written in the form

$$
(\Delta \mathrm{p} / \Delta \mathrm{l})_{\ell}=(\Delta \mathrm{p} / \Delta \mathrm{l})_{\mathrm{g}} \varphi_{\mathrm{tt}}
$$

where $\varphi_{t t}$ is an empirical function of the dimensionless Martinelli parameter $x_{t t}$ :

$$
\mathrm{X}_{\mathrm{tt}}=\left(\mu_{\ell} / \mu_{\mathrm{g}}\right)^{0.1}\left(\rho_{\mathrm{g}} / \rho_{\ell}\right)^{0.5}\left(\frac{1-\mathrm{x}}{\mathrm{x}}\right)^{0.9}
$$

The dynamic viscosity is $\mu$, and $\rho$ is the density of the gaseous or liquid phase, respectively; $\mathbf{x}$ is the quality of the gas flow. (For further details, References 192 and 186 should be consulted.)

Many modifications have since been proposed to improve the original Martinelli correlations. Baroczy ${ }^{(193)}$ recently introduced a modified correlation which seems to hold for water and for liquid metals. However, substantiating experimental evidence is rather scarce to date, and more reliable test data for liquid metal two-phase flow are needed.

A correlation for liquid fraction using Martinelli parameters, has also been proposed by Baroczy. (194) Within an error span of about $\pm 50 \%$ it supposedly holds both for water and for liquid metals. 
It has also been shown that the critical heat flux for water and for liquid metals follows essentially a common correlation, $(195,186)$ although it is unlikely that this can be used in any practical application at this time because the reported correlation is in dimensional form only.

Even though such common correlations are sometimes quite helpful in predicting overall liquid metal heat transfer coefficients from water tests, their limitations should be kept in mind:

1) A change in the wettability of the liquid metal may change the predicted heat transfer coefficient by as much as a factor of 10 .

2) It cannot necessarily be expected that extrapolations beyond the range of existing test data will hold true.

3) Even if the $\mathrm{Nu}$-number can be derived for liquid metals, the many factors that make up the number still remain unknown.

4) Even for the experimentally obtained test data the error spread is quite large (up to 50\%) and even these data are sometimes challenged by other investigators.

A rather cautious approach is still warranted, therefore, if liquid metal two-phase flow and heat transfer are to be simulated in water tests. ${ }^{(55)}$ Only additional test data and further progress in theoretical under standing will ultimately show to what extent such water tests are practically useful.

\section{SUMMARY ON HEAT TRANSFER}

Apparently, quantitative liquid metal heat transfer data cannot be obtained from water tests. Hydraulic data (such as pressure drops) can be obtained as usual under small and moderate heat transfer conditions. Scale-model testing with liquid metals at low temperatures (by simulating Pe-numbers) is justified as a first approximation to obtain full-scale heat transfer data for liquid metals at higher temperatures. Deviations must be expected; their magnitude will vary from case to case and they are not generally determinable. Because of the experimental difficulties involved in heat transfer measurements, test data by it self is normally accurate only within $\pm 15 \%$. 


\section{SPECIAL FLOW PROBLEMS AND THEIR MODEL TESTING}

Besides the major fluid mechanical component problems that have been considered in the previous sections, there are several minor problems which could occur in liquid metal flows. Such problems are not necessarily connected with a particular flow component but could arise in a variety of different circumstances. This section is an examination of the way in which such liquid metal flow problems as cavitation, vortexing, etc., could be investigated by water model testing.

\section{A. CAVITATION}

Besides occuring in pumps, cavitation can occur in various other flow devices: valves, ${ }^{(196)}$ orifices, nozzles, $(197,198)$ bearings, $(96,97,199)$ etc. Cavitation always starts in flow areas where the local velocities $u$ are so high that the corresponding static pressures p decrease below the vapor pressure $\mathrm{p}_{\mathrm{v}}$ of the liquid. A nondimensional pressure ratio $\sigma$, made up of these parameters, has proven quite useful as a criterion for determining the existence of cavitation. The cavitation number $\sigma$ is defined as

$$
\sigma=\frac{p_{\infty}-p_{v}}{(\rho / 2) u_{\infty}^{2}},
$$

where $p_{\infty}$ and $u_{\infty}$ refer to convenient reference pressures and velocities (e.g., in undisturbed parallel flow). When $\left(p_{\infty}-p\right) /(\rho / 2) u_{\infty}^{2}$ becomes of the same order of magnitude as $\sigma$, cavitation is likely to set in. Each particular flow configuration has its characteristic $\sigma$.

As long as $p_{v} \ll p, \sigma$ can be approximated simply by $\sigma \approx \mathrm{p}_{\infty} /(\rho / 2) \mathrm{u}_{\infty}^{2}$. So that to avoid cavitation in a particular case (e.g., flow through a certain type of valve) the static pressure $p_{\infty}$ in the incoming undisturbed flow must not be lowered so far that $\left(p_{\infty}-p\right) /(\rho / 2) u_{\infty}^{2}$ drops below its critical value $(\alpha)$ for this particular flow situation. In a closed system (as in a test loop) $\mathrm{p}_{\infty}$ can usually

*Because avoiding cavitation is of primary importance to the efficient operation of pumps, its special aspects with respect to pump testing a re treated in detail in Section $\mathrm{V}$. 
be changed independently from $u_{\infty}$ by increasing or decreasing the cover gas pressure. For example, if the limit for the onset of cavitation is $\sigma=0.5$, the velocity head of the flow [dynamic pressure $(\rho / 2) u_{\infty}^{2}$ ] must not be larger than twice the static pressure to avoid cavitation. However, from theoretical flow analysis the cavitation number $\sigma$ can only be estimated on a very approximate basis. A reliable determination must be done experimentally.

Since the $\sigma$ for the onset of cavitation depends primarily on the geometry of the flow, it is possible to use the same $\sigma$, which has been determined e.g., in a water model test, for an equivalent flow situation in liquid metals also. If the vapor pressures $\mathrm{p}_{\mathrm{v}}$ of the water and the liquid metal are both small compared to the reference pressure $p_{\infty}$, they can be disregarded in a first approximation in both cases. [Otherwise they must be considered according to the original definition of $\left.\sigma=\left(p_{\infty}-p_{v}\right) /(\rho / 2) u_{\infty}^{2} \cdot\right]$ For greater accuracy it should be possible (through the judicious choice of operating temperatures) to make the vapor pressures equal. For example, $1200^{\circ} \mathrm{F}$ sodium and $104^{\circ} \mathrm{F}$ water have the same vapor pressure, 1.1 psia. However, not too much is really gained by this procedure as far as accuracy of model testing is concerned. Moreover, it complicates the test. As pointed out before, the onset of cavitation is primarily determined by the highly localized peak velocities and not by the average velocities. Unfortunately only the latter can normally be determined by theoretical analysis.

The ratio of the peak velocities to the average velocities depends very much on the details of the geometric flow boundaries (whether an edge has a sharp corner or is slightly rounded, etc.) and also on the Re-number of the flow. Additionally, the onset of cavitation is influenced by a number of secondary parameters (such as gas content of the liquid, concentration of solid contaminants in the liquid, etc.) which are relatively hard to determine on a quantitative basis.

For cavitation model testing the following rules should be observed:

1) The (water) model should be a very exact replica of the (liquid metal) prototype and preferably full-scale.

2) A water velocity should be chosen so that the model Re-number equals the prototype Re-number. 
3) A water temperature should be chosen so that kinematic viscosities and vapor pressures are of comparable magnitude for the model and for the prototype. (It will generally be impossible to simulate both viscosity and vapor pressure exactly.)

4) The water used for model testing should be very clean and free from entrained air.

The results of such water model tests allow conclusions of the following kind:

1) Where there is clearly no cavitation in water, there will also be no cavitation in liquid metals for the same $\left(p_{\infty}-p\right) /(\rho / 2) u_{\infty}^{2}$.

2) Where the water tests definitively show the existence of cavitation, the liquid metal prototype is likely to cavitate also for the same $\left(p_{\infty}-p\right) /(\rho / 2) u_{\infty}^{2}$.

3) By varying $p_{\infty}$ and/or $u_{\infty}$ in the water model test it is usually possible to arrive at $a\left(p_{\infty}-p\right) /(p / 2) u_{\infty}^{2}$ for which cavitation will set in. At approximately the same value the liquid metal prototype will also start cavitating.

In such borderline cases (where it is also experimentally quite difficult to reliably prove or disprove the existence of any cavitation), it is not certain to what extent conclusions from water tests can be applied with confidence to liquid metals. It is likely that in these cases only a test of the prototype itself will provide a reliable answer.

As far as cavitation damage is concerned, no general correlation has been found between water and liquid metals, ${ }^{(198)}$ and it is suspected that such correlations do not in fact exist. Recently new attempts have been made to find such correlations empirically. (200) However, the results of these investigations still appear to be ambiguous.

\section{B. VORTEXING}

Liquid rotation and vortexing (such as occurs while draining a vessel filled with a liquid) pose special flow problems which often assume major proportions in certain nuclear engineering applications. For liquid metals such vortexing must always be avoided because it usually involves gas entrainment, which is highly undesirable. 
The fluid mechanics of liquid rotation and vortexing are intrinsically very complex and no convincing and comprehensive theory concerning these phenomena is available, in spite of many serious attempts $(201-205)$ to develop such a theory. Model testing and previous experience are practically the only means of obtaining any reliable design information. There have been attempts to define similarity parameters for vortex motion, ${ }^{(206)}$ but they are still controversial and will not be discussed here.

Water can be substituted for liquid metal in such experiments if the kinematic viscosity is duplicated, as it is generally assumed that the viscous boundary layers at the bottom of the vessel influence the generation of outflow vortexes." But regardless of whether experiments are performed with water or with the liquid metal, the results are often inconclusive because the probability for the development of a vortex depends to a very large degree on the amount of circulation ${ }^{\dagger}$ present in the fluid when a drain cycle is being started. It furthermore depends on any rotation induced by secondary flow effects during the draining process itself. It is very difficult to measure or control such initial and induced circulation, since it usually is originated by many different interacting sources. For the same reason it is also very difficult (if not impossible) to simulate them in a model test. Such contributing sources include residual circulation remaining from a previous cycle, circulation generated during the filling process of the vessel, circulation generated by flow due to free convection, circulation generated by the inflow of any gas or fluid which replaces the out-flowing liquids, circulation originating from forces due to earth rotation (Coriolis forces), circulation generated by certain vibrations of the vessel, etc. It is also quite possible that quantities such as the turbulence level and the viscosity (as distinct from the Re-number) also play an important role. (207)

In many practical vortexing problems, however, the test objective is merely the evaluation of the effectiveness of vortex suppressing devices such as grids

\footnotetext{
"Quite frequently such vortexes are called bathtub vortexes. TThe circulation $\Gamma$ over an area $S$ is defined as $\Gamma=\int \operatorname{curl} \vec{v}$ dS where $\vec{v}$ is the velocity vector of the flow. Similarly the total circulation contained in a volume $V$ is $\Gamma=\int \operatorname{curl} \overrightarrow{\mathrm{V}} \mathrm{dV}$. As the local distribution of $\Gamma$ is almost always subject to random statistical fluctuations, the value of $\Gamma$ will depend on the summation of such random contributions and will change accordingly.
} 
or baffles. Such a test is usually performed for worst conditions, which can be anticipated for the prototype arrangement. To this end a certain degree of initial circulation is artificially induced at the beginning of the drain cycle. If a vortex suppressor clearly prohibits the development of a bathtub vortex in the water model test, it can be assumed with high confidence, that it would also be effective for liquid metals in the same configuration. This is particularly true if the tests are performed at (or nearly at) full scale. The effects of any differences in viscosity and density between liquid metal and water are likely to be small compared to the effect of initial and induced circulation in the fluid, especially if water temperatures have been chosen such that the property differences are at a minimum. The higher an initial circulation can be made in such water tests without the subsequent appearance of a bathtub vortex, the more effective is the vortex suppressing device. This can be carried so far, that it can finally be concluded beyond any reasonable doubt, that an equivalent device would also be effective for all practical purposes in the liquid metal prototype.

In cases of marginal model performance, only the prototype itself will give the final answer. And even then it is likely that under such conditions the answer will still be somewhat ambiguous, as it may happen that a vortex will develop only in a certain percentage of cases because of the statistical distribution of the fluid circulation.

\section{VALVES AND FLOW METERS}

Valves and flow meters $(208,209)$ both represent geometrical constrictions in the flow path. Such constrictions, as well as any following enlargements, can be smooth (as in a Herschel-Venturi and a diffusor), or they can be abrupt (as in an orifice or valve). In most cases they are a combination of these two extremes.

Because it is quite difficult, if not impossible, to determine the pressure loss* through a constriction (such as a partially opened valve) on purely analytical

*The total pressure loss is usually made up of pure frictional losses and of Borda-Carnot losses due to flow separation, cavitation, etc. In a welldesigned Herschel-Venturi meter the pressure loss may be almost completely due to friction, whereas in an orifice meter wall friction may represent only a few percent of the total loss. 
grounds, the experimental testing of valves and flow meters could be quite important. $(67)$.

In particular, it is usually necessary to determine the mass flow rate $Q$ as a function of the pressure difference $\left(p_{u}-p_{d}\right)$ in the flow upstream and downstream of the restriction (area A). This relation is commonly expressed analytically in the following form:

$$
Q=C_{d} A \sqrt{\frac{2\left(p_{u}-p_{d}\right)}{\rho}}
$$

The dimensionless discharge coefficient $C_{d}$ is a function of the particular geometry of the constriction. It is largely independent of Re-numbers (especially if Re-numbers are large), and it is possible, therefore, to substitute water for liquid metals in a model test as long as the geometric details of the constriction are identical. (This is most certainly assured if the actual full scale liquid metal prototype is being tested in water.) Even such apparent details as to whether an edge is sharp or slightly rounded may have unpredictable effects. As pointed out before, it is also important that the incoming flow in the model has the same distribution as in the prototype, which means that elbows upstream in the flow, for example, may have to be simulated also (see page 21$)$.

The Borda-Carnot loss of a sudden enlargement in a flow duct (sometimes also called shock loss), as generally derived in textbooks, $(36,46)$ is theoretically independent of viscosity (or Re-number). The pressure loss $\Delta p$ in form of the Euler number is given by $\Delta p /(\rho / 2) u^{2}=1-\left(A_{1} / A_{2}\right)$, where $A_{1}$ and $A_{2}$ are the areas of the two different cross sections of the duct. This means that experiments performed in water should yield the correct friction coefficients for equivalent flow in liquid metals. A closer examination ${ }^{(46)}$ reveals however, that this is essentially true only for the turbulent regime ( $\operatorname{Re}>3500$ ) and also only for flows with a uniform velocity distribution. In the transitional and laminar regime, the pressure loss can increase substantially above this value; however, the increase is not monotonic with decreasing Re-number. Furthermore, it depends on $A_{1} / A_{2}$. A nonuniformity in the velocity distribution of the incoming flow also has the effect of increasing the pressure loss. 
It can be expected that essentially the same arguments apply to any general flow condition which involves flow separation. Therefore, in practice many types of flow (such as might occur in valves, elbows, pumps, etc.) will be affected by these considerations. Unfortunately, the whole problem of flow separation is highly complex $(210)$ and it is very doubtful whether the free stream $\mathrm{Re}-\mathrm{number}$ is really the most important parameter which determines separation. Boundary layer theory is not yet sufficiently advanced to provide a set of nondimensional parameters (either derived by dimensional analysis or by differential similarity) which would allow modeling of boundary layer separation in a quantitative way for the general case of three-dimensional boundary layers.

For the time being, therefore, it remains uncertain exactly to what extent wate $r$ model experiments can be used in a quantitative way to predict liquid metal performance where the main flow is affected appreciably by separation phenomena. However, as a first approximation such model tests are well justified.

As pressure losses in flow constrictions can be quite large, it sometimes happens that the static pressure drops below the vapor pressure of the liquid, in which case cavitation is apt to occur. However, as described in more detail in Section IX. A, it is possible to obtain certain experimental information on the existence of cavitation if water is substituted for liquid metals in a model test.

Calibration of a liquid metal flow meter in a water model test is justified if only a limited accuracy (not better than about $5 \%$ ) is required. Calibration accuracy can generally be improved by improving the uniformity of the flow, (209) by a high degree of geometrical similitude (preferably full scale), by avoiding any cavitation, $(197,198,211,212)$ and by improving pressure measuring techniques, etc. It appears that by very careful full-scale prototype calibration an ultimate accuracy limit of about $1 \%$ can be reached. (67)

\section{FLOW TRANSIENTS}

This section discusses the question of whether water tests would also yield any meaningful information with respect to unsteady flow phenomena that could occur in liquid metal flows. 
The dynamics of fluid transients ${ }^{*}$ in engineering systems is by itself another of the very specialized and complicated subjects of fluid mechanics. $(213-215)$ Because the present knowledge in this field (especially that of turbulent unsteady flow) is still quite fragmentary, $(216,217)$ model test techniques would be highly desirable. However, the experience and general rules needed to translate the results from model tests to meaningful engineering design information are still widely lacking.

Besides the unusual flow parameters (such as Re-numbers) which must be simulated for steady flow, there are a number of other parameters ${ }^{\dagger}$ which have special significance for unsteady flows only. The most important of the fluid properties in this respect is the compressibility of the fluid (which is the inverse of the liquid bulk modulus). It happens that the compressibility of water is about twice as high as that of liquid sodium. Although absolute pressure and temperature do have some influence on the compressibility, this ratio does not change much until the critical temperatures $\left(374^{\circ} \mathrm{C}\right.$ for $\mathrm{H}_{2} \mathrm{O}$ and $\sim 2500^{\circ} \mathrm{C}$ for Na) are approached.

The compressibility and the density of a liquid determine its sound velocity $c$ (i.e., the velocity by which pressure transients propagate). For water at $20^{\circ} \mathrm{C}$ $\left(68^{\circ} \mathrm{F}\right)$ the sound velocity is $4720 \mathrm{ft} / \mathrm{sec}$. For liquid sodium at $650^{\circ} \mathrm{C}\left(1200^{\circ} \mathrm{F}\right)$ the sound velocity is $8850 \mathrm{ft} / \mathrm{sec}$. In closed conduits (such as pipes) the effective sound velocity "a" can be substantially smaller than the se values, depending on the elastic properties and on the stress factor $f$ of the pipe walls ( ${ }^{217}$ ) Although elastic constants are a function of temperature it is still possible, through the judicious choice of the stress factor $f$, to make the effective wave velocity "a" for cold water flow in one pipe equal to that for hot liquid metal flow in a nother pipe.

*The subject as considered here shall include not only single events, such as surges and waterhammer, but also repetitive phenomena such as vibrations caused by turbulent flow, etc.

†These parameters are still dimensional and it appears that the usual methods of dimensional analys is have not yet been applied extensively to the field of nonstationary fluid mechanics. 
In a quantitative way "a" can be determined from the following formula:

$$
a=\frac{c}{\sqrt{1+f(B / E)}}
$$

where

$$
\begin{aligned}
\mathrm{c}= & \text { sound velocity in the liquid having infinite dimensions } \\
\mathrm{B}= & \text { bulk modulus of the liquid } \\
\mathrm{E}= & \text { modulus of elasticity of the pipe material } \\
\mathrm{f}= & \text { stress factor of the pipe (For thin-walled pipes } \mathrm{f}=\mathrm{D} / \mathrm{e}, \mathrm{D} \text { being } \\
& \text { the diameter and } \mathrm{e} \text { the wall thickness of the pipe). }
\end{aligned}
$$

For water at $65^{\circ} \mathrm{F}$ the bulk modulus is approximately $2 \times 10^{4}$ atm, whereas for liquid sodium at $1200^{\circ} \mathrm{F}$ it is approximately $4 \times 10^{4} \mathrm{~atm}$. The modulus of elasticity of a steel pipe at $65^{\circ} \mathrm{F}$ may be of the order of $2 \times 10^{6}$ atm whereas this figure might drop approximately by a factor of 3 to $2 / 3 \times 10^{6}$ atm for a temperature of $1200^{\circ} \mathrm{F}$. (The cited figures change with the particular type of material.) For example, if a stress factor of $f=50$ would be selected for a sodium pipe (steel) operating at $1200^{\circ} \mathrm{F}$ the effective wave velocity "a" would be $4425 \mathrm{ft} / \mathrm{sec}$. To obtain the same velocity in a steel pipe carrying water at $65^{\circ} \mathrm{F}$ a stress factor of $\mathrm{f}=14$ would be required. In which case, a steel pipe of 30 -in. diameter with a 0.6 -in.-thick wall for sodium flow at $1200^{\circ} \mathrm{F}$ is equivalent to a steel pipe of 10-in. diameter with a 0.7 -in.-thick wall for water flow at $65^{\circ} \mathrm{F}$, as far as the propagation velocity of fluid transients is concerned.

If the amplitudes of flow transients are also to be simulated, the flow velocities $V$ must be equalized. According to Joukowsky, $(218,87)$ the maximum pressure rise (due to a sudden closure of a value, for instance) in a pipe is $h_{\max }=a V_{o} / g$, where $V_{o}$ is the fluid velocity before the closure. For a sudden partial valve closure the pressure rise is $\mathrm{h}=\mathrm{a} \Delta \mathrm{V} / \mathrm{g}$, where $\Delta \mathrm{V}$ is the change in velocity due to the different valve setting. A sudden opening of a valve is accompanied by a transient pressure decrease on the upstream side which cancause cavitation. If the change in the valve setting takes a finite time, more elaborate computations (and/or graphical methods) must be applied to obtain the amplitudes and phases of the transients. For such more realistic cases model tests would 
be very helpful and, as shown in the preceeding, both sound and fluid velocities (a and V) must be simulated in order to model any liquid metal transients in a water test.

It should be noted that the requirement for the simulation of "a" puts a restriction merely on $f$ and not on the absolute value of the pipe diameter $D$. Therefore, it is still theoretically possible to choose a D such that the Renumbers also become equal. However, this would often lead to rather extreme and impractical requirements such that, e.g., to simulate a 30-in. diameter sodium pipe, the water pipe diameter would have to be 150 in. The wall thickness of this water pipe would have to be $3.5 \mathrm{in}$.

Another important factor to be considered in the evaluation of fluid transients is the travel time $T=L / a$ of a pressure wave. Since $L$ is the length of the pipe, $T$ can be kept the same for the model and the prototype only if model and prototype pipes are of equal length (under the assumption that "a" is to be duplicated also).

Still a nother important scaling factor for transients in a pipeline is its characteristic impedance $Z_{0}=\rho a / A$, where $A$ is the cross-sectional areas of the pipe. If this parameter is duplicated it puts a restriction on $A$ (or the diameter D), usually with the result that the Re-number can no longer be duplicated.

Flow transients as they occur in nuclear engineering applications are usually not restricted to temporal changes in pressures or volume flow but frequently they are also accompanied by thermal transients (such as period spikes in reactors)."

Since it is highly impractical to fully simulate liquid metal flow transients by water model tests for an isothermal case, the inclusion of thermal excursions appears to be impossible.

Whereas the opening or closing of a valve usually results in a single transient which dies out fairly rapidly, lasting oscillations or vibrations $(219-221)$ in a piping system can be set up either by oscillatory fluctuations (e.g., in a

* Such thermal transients are mostly due to the close interaction between fluid flow and reactor response (e.g., due to a gas bubble). 
pump discharge) or they can be self excited (due to vortex generation for example). A piping system normally has a number of resonant frequencies and it can happen that vibrations sometimes become untolerable if the existing oscillations are in tune with the resonant frequencies. $(222,223)$ Because it is practically impossible to simulate the complicated interplay of the various exciting and resonating frequencies in a model test, even under equal temperature conditions, successful modeling of a hot liquid metal loop with a water loop is practically impossible in this respect. If vibrations are detected in a prototype system, attempts are usually made to find the sources of these vibrations. Once they have been determined it is often possible to eliminate them. Sometimes vibrations occur in a highly localized area only. For example, it is possible that certain rods or tubes in reactor cores or heat exchangers vibrate due to fluid flow action. This can happen in cross flow as well as in parallel flow. $(224,225)$ In such cases it might be possible to investigate this particular device (or part of a device) in a model test. If it is possible, for instance, to eliminate the vibrations in a water test by a certain change in the geometrical configuration, it is likely that a similar change will also be effective in the corresponding liquid metal case. As this is mostly a trial and error method, the water model can give only qualitative results. When reactor cores are tested hydraulically to determine their flow characteristics, they are also usually instrumented with vibration transducers to detect any serious vibrations. If the tests show that the cores are essentially free from vibrations it is as sumed that they would not vibrate under operating conditions with liquid sodium. In several practical cases such an assumption has been verified later by the actual performance in the reactor. However, model tests have not been used extensively, to date, for testing fluid transients, not even in water systems. Therefore, very little practical experience is pres ently available in this field. Not a single case of testing fluid hammer type transients was found in the literature on liquid metals.

Although it appears theoretically possible to use carefully selected water models to simulate transients that might occur in a liquid metal loop, the scaling requirements are such that a practical realization of such model tests is almost impossible. In many cases their cost might in fact exceed the cost of actual fullscale prototype testing. It appears that oscillations and vibrations generated by liquid metal flow can be tested in water models in a qualitative way only. 


\section{$X$. CONCLUSIONS AND RECOMMENDATIONS}

In the course of this study, various possibilities suggested themselves (usually only implicitly) as workable approaches to be followed in a particular case where liquid metal problems are to be solved by model testing. In the following text a summary of general recommendations ${ }^{*}$ is given in a more explicit form. (Unfortunately, the subject is too complex to be covered by two or three sentences.)

Once it has been decided that a particular liquid metal flow problem ${ }^{\dagger}$ should be investigated by some kind of a model test, the following questions should first be resolved:

1) Does this particular problem lend itself to a successful water test?

2) What will be the results and approximate accuracies and what are the limitations in such a test?

3) What should the scale of the model be?

4) What will be required to accomplish the desired test objectives (in time, money, etc.)?

As unequivocal answers to all these questions a re often not obtainable, a compromise solution should be selected with respect to the various conflicting requirements.

Each problem should be individually considered in this respect. The fact that a water model test has worked well in one instance is no cogent reason that it would work equally well in a different case.

Every new approach to a model test should also be justified by specific reasoning. Statements such as "principles of dimensional analysis are being applied for prediction of hydraulic characteristics" should be avoided as being too general.

\footnotetext{
*Additional specific recommendations with regards to the various flow components are given at the end of the respective chapters.

tThis also includes the mere experimental verification of a new analytical design.
} 
A model test should never be run for its own sake, or just because it has become a custom to run a model test for a certain application. It should be disapproved when it takes almost the same effort as to run a test with the actual full-scale prototype.

If a model test is being performed it often takes only a little extra effort to obtain additional data which might be very useful for further development or verification of a new theory. Such data should be recorded even if they are of no real importance to the current test objective.

Fluids (such as acetaldehyde) with properties closer to those of liquid metals should be preferred over water in tests where a high degree of accuracy is important.

If a completely new fluid flow theory is to be verified (e.g., theory of tur bulent hydrostatic lubrication) water tests are usually adequate.

If the compatibility and/or endurance of certain materials with hot liquid metal flows are to be investigated water modeling is obviously useless.

High temperature effects or problems connected with thermal transients and thermal gradients cannot be investigated in water; only the full-scale prototype would give reliable answers.

It is sometimes important to faithfully simulate not only certain geometrical details of the flow boundaries but also the velocity distribution of the incoming flow.

It often pays to search the literature for previous similar tests, if only to find out what pitfalls and errors should be avoided.

If phenomena are so complicated that no adequate theory exists, or if the correct choice of characteristic nondimensional parameters is in doubt, it is nevertheless often possible to find ${ }^{*}$ correlations to which the test data can be fitted. This usually brings some kind of order to an otherwise confusing amount of data. But water tests should be substituted for liquid metal tests only if it

*This could be done more or less intuitively, or by trial and error and/or curve fitting, etc. 
has been shown experimentally that the same type of correlation holds for water and for liquid metals. Reliable predictions can be made only over the limited range for which correlations have been established by experiments. Extrapolations out of this range are often not justified and should be avoided.

While trying to decide between a test performed in water or in the real fluid (hot liquid metal) it should be kept in mind that instrumentation for water tests is readily available and is usually rather accurate. This is often not the case for liquid metal instrumentation. Therefore, not much may be gained by doing a certain test in liquid metals instead of in water.

In many practical cases a cursory test in a water model may be all that is needed. There shouldn't be any hesitation about performing such a test as long as the limitations with regard to overall accuracy are considered.

The degree of error involved in the results and predictions derived from a water test and applied to liquid metals cannot be accurately stated. Since figures would depend on many particulars they would differ from case to case. In general, a lower limit on such errors might be on the order of a few percent. The upper error limit is much harder to estimate; in rare cases, predictions could be off by as much as a factor of 2 or 3 . 


\section{REFERENCES}

1. M. D. Hersey, Theory and Research in Lubrication, (John Wiley and Sons, New York, 1966)

2. H. L. Langhaar, Dimensional Analysis and Theory of Models, (John Wiley and Sons, New York, 1951)

3. A. A. Gukhman, Introduction to the Theory of Similarity, (Academic Press, New York, 1965)

4. L. I. Sedow, Similarity and Dimensional Methods in Mechanics, (Academic Press, New York, 1959)

5. A. Betz, "Ähnlichkeitsmechanik and Modelltechnik" in Hütte, Vol. I (Akad. Verein Hutte, Berlin, 1955) p 744-752

6. S. J. Kline, Similitude and Approximation Theory (McGraw-Hill Book Co. , New York, 1965)

7. M. Holt, "Dimensional Analysis," Handbook of Fluid Dynamics

(V. L. Streeter, ed. McGraw-Hill Book Co., New York, 1961)

8. P. W. Bridgman, Dimensional Analysis (Yale University Press, 1937)

9. J. Serrin, Mathematical Principles of Classical Fluid Mechanics, Encyc. of Phys., Vol. VIII, l (Julius Springer, Berlin, Göttingen, Heidelberg, 1960) p. $181-183,243-246$

10. K. Oswatitsch, Physikalische Grundlagen der Strömungslehre, Encyc. of Phys. Vol. VIII, l (Julius Springer, Berlin, Göttingen, Heidelberg, 1960) p. $56-61$

11. P. J. Berenson, 'Flow Stability in Multitube Forced Convection Vapor izers, "APL-T DR-64-117 (1964)

12. B. Gebhart, Heat Transfer (McGraw-Hill Book Co., New York, 196 l)

13. J. A. Barker, Lattice Theories of the Liquid State (MacMillan, New York, 1963)

14. J. O. Hirschfelder, C. F. Curtiss, and R. B. Bird, Molecular Theory of Gases and Liquids (John Wiley and Sons, New York, 1964)

15. M. Born and H. S. Green, A General Kinetic Theory of Liquids (Cambridge University Press, New York, 1944)

16. J. A. Pryde, The Liquid State (Hutchinson, London, 1966) 
17. H. S. Green, Structure of Liquids, Encyc. of Phys. Vol X (Julius Springer, Berlin, Göttingen, Heidelberg, 1960)

18. J. D. Bernal, "The Structure of Liquids," Proc. Roy. Soc. A 280, p 299$322(1964)$

19. L. E. Ballentine and V. Heine, "On a Theory of Liquid Metals," Phil. Mag. 9, p 617-622 (1964)

20. F. Franks and W. Good, "Mechanism of Viscous Flow in Water," Nature 210, p 85-86 (1966)

21. "Symposium of Ice, Water, and Aqueous Solutions," Developments in Applied Spectroscopy Vol. 6 (Plenum Press, New York, 1967)

22. C. C. Addison, "Liquid Sodium," Endeavour 26, p $91-95$ (May 1967)

23. P. D. Adams, et al. (eds.), Proceedings of the International Conference on the Properties of Liquid Metals, Advances in Physics, p 147-748 (1967) (also Taylor and Francis Ltd., London, 1967)

24. J. R. Wilson, "The Structure of Liquid Metals and Alloys," Metallurg. Rev. 10, No. 40, pp 385-590 (1965)

25. B. Widom, "Intermolecular Forces and the Nature of the Liquid State," Science 157 p $375-400$ (1967)

26. R. D. Miller et al., "Liquid Metals Handbook, Sodium-NaK Supplement," TID 5277 (1955) (New edition in preparation)

27. K. H. Spiller, "Zur Kenntnis der physikalischen Stoffeigenschaften von Flüssigmetallen," Atomkernenergie 10, p 127-138; 215-227 (1965)

28. G. H. Golden and J. V. Tokar, "Thermophysical Properties of Sodium," ANL-7323 (1967)

29. N. E. Dorsey, Properties of Ordinary Water-Substance (Reinhold Publishing Corp., New York, 1940)

30. C. D. Hodgman, ed., Handbook of Chemistry and Physics, 48th Ed. (Chemical Rubber Publishing Co., Cleveland, 1967)

31. K. D. Hayden, "Survey of Hydraulic Modeling Experience for Reactor Core Systems," BNW L 518 (1967)

32. L. Prandtl and O. G. Tietjens, Fundamentals of Hydro-and Aeromechanics (Dover Press, New York, 1957) 
33. A. Betz, Konforme Abbildung (Julius Springer, Berlin, New York, 1964)

34. J. Ackeret, "Aspects of Internal Flow," Fluid Dynamics of Internal Flow, (G. Sovran, ed., Elsevier Publishing Co., Amsterdam, London, New York, 1967) pp 1-26

35. D. S. Trent, "Applications of Geometric Models for the FFTF Hydraulic Core Mockup," BNW L 575 (1967)

36. L. Prandtl, Führer durch die Strömungslehre, 6th Ed. (Vieweg, Braunschweig, 1965)

37. A. Pope and J. J. Harper, Low Speed Wind Tunnel Testing, (John Wiley and Sons, New York, 1966)

38. J. Flanigan and A. A. Putnam, "The Use of Fluid Dynamic Modeling Techniques," Batelle Tech. Rev., pp. 1-7 (February 1964)

39. R. O. Sandberg, "Analysis of CVTR Thermal-Hydraulic Performance at 65 Mw Operation, "CVNA 286 (1967)

40. H. Ito, "Friction Factors for Turbulent Flow in Curved Pipes," (Trans. ASME) J. Basic Eng. 81, p 123-134 (1959)

41. H. Richter, Rohrhydraulik, 4th Ed. (Julius Springer, Berlin, Göttingen, Heidelberg, 1962)

42. B. Eck, Technische Strömungslehre, 5th Ed. (Julius Springer, Berlin, Göttingen, Heidelberg, 1957)

43. H. Schlichting, Boundary-Layer Theory, 4th Ed. (McGraw-Hill Cook Co., New York, 1960)

44. R. W. Detra, "The Secondary Flow in Curved Pipes," Mitt. Inst. Aero. E.T.H., Zürich, No. 20 (1953)

45. W. R. Hawthorne, "The Applicability of Secondary Flow Analysis to the Solution of Internal Flow Problems," Fluid Dynamics of Internal Flow (G. Sovran, ed., Elsevier Publishing Co., Amsterdam, London, New York, 1967) p 238-269

46. I. E. Idel'chik, "Handbook of Hydraulic Resistance," AEC-tr 6630 (1966)

47. R. J. Begley, "Hydraulic Characteristics of HNPF-8 - Rod Fuel Element," NAA-SR - 7475 (1963)

48. M. A. Perlow, "SNAP-2 Primary Coolant Development," NAA-SR-6439 (1961) 
49. J. H. Brindley, "Hydraulic Studies of the SRE-PEP Core III Process Channel," NAA-SR -1 1163 (1965)

50. D. A. Huber, "Core Hydraulics," Organic Reactor Heat Transfer Manual, NAA-SR -Memo-7343 (1962)

51. J. G. Yevick (ed.), Fast Reactor Technology: Plant Design (Massachusetts Institute of Technology Press, 1966)

52. C. F. Bonilla, Nuclear Engineering, (McGraw Hill Book Co., New York, 1957)

53. G. Hetsroni, "Use of Hydraulic Model in Nuclear Reactor Design," Nucl. Sci. and Eng. 28, p 1-11 (1967)

54. L. J. Flanigan et al., "Model Studies of Flow in PWR Core 2 Fuel As semblies," BMI 1720 (1965)

55. J. O. Smith et al., "Model Studies of Reactor Coolant and Fuel Pin Behavior under Conditions of Coolant Boiling and Overheating of the Fuel Elements," TID-18229 (1961)

56. K. G. Eickhoff, J. Allen, and C. Boorman, "Engineering Development for Sodium Systems," London Conference on Fast Breeder Reactors, BNES $66-5 B / 5(1966)$

57. J. F. W. Bishop et al., "Design and Development of Fuel and Fuel Elements for Prototype Fast Reactor," London Conference on Fast Breeder Reactors, BNES-66-4B-1 (1966)

58. A. E. Perry, "The Concept of Hydraulic Diameter," ASME 64-W A-FE 31 (1964)

59. B. W. Le Tourneau et al., "Pressure Drop for Parallel Flow Through Rod Bundles," Trans. ASME 79, p 1751 (1957)

60. R. T. Berringer and A. A. Bishop, "Model Study of the Pressure Drop Relationships in a Typical Fuel Rod Assembly," YAEC-75, Westinghouse Electric Corp. (February 1959)

61. O. E. Homeister et al., "Model Studies of the Flow Characteristics of the Fuel Elements for the Enrico Fermi Reactor, "Fourth Nuclear Engineering and Science Conference, Chicago, March 17-21, 1958, 621, 48 N883

62. A. C. Spengos, "Test on Models of Nuclear Reactor Elements - IV Model Study of Fuel Element Supports," UMRI 2431-4-P, University of Michigan (July 1959) 
63. A. N. de Stordeur, "Drag Coefficients for Fuel Element Spacers," Nucleonics 19, No.6, p 75-79 (June 1961)

64. J. J. Edwards et al., "Cylindrical Fuel Element Flow Stability Program," APDA - 181, (1967)

65. R. A. Dean, "Coolant Mixing in Open Lattice Reactor Cores," WCAP-3735, Westinghouse (August 1963)

66. J. H. Brindley et al., "Vibration Studies of the Large SGR Fuel Element and Process Channel," NAA-TDR-9164 (1964)

67. R. J. Begley, "Flow Measurement with Orifice and Venturi Meters in Large Diameter Piping for Sodium Cooled Reactors," NAA-SR-Memo-5859 (1960)

68. D. T. Rymsa and G. P. Wachtel, "Flow Model Studies for the EBR-II," TID-21953 (1961)

69. V. I. Subbotin et al., "Hydraulic Resistance of Narrow Annular Channels with Helical Pins," Sov. Atom. Energy 21, p 626-629 (1966)

70. J. A. Hagel, "Hydraulic Characteristics of HNPF 19-Rod Fuel Element," NAA - SR - 5340 (1960)

71. H. Vermij, "Measurement of Pressure Drop Across the Supporting Grids of a Sodium Heated Steam Generator," SR - TN/6610-03 (1966)

72. R. Möller, "Heat Transfer and Pressure Drop on Rod Bundles with Axial Flow, "EURFNR-257L (1966)

73. S. L. Dixon, Fluid Mechanics, Thermodynamics of Turbomachinery (Pergamon Press, New York, 1966)

74. J. Baumier and H. J. Gallion, "Mechanical Pumps for Liquid Metals," AEC-TR -6354 (1963)

75. L. F. Bowles and D. Taylor, "The Sodium Pumps for the PFR," Nucl. Eng. (May 1967)

76. C. Ferguson et al., "Final Report on Development of LCRE Liquid Metal Pumps and Sumps," PWAC 386 (1965)

77. H. W. Savage and A. G. Grindell, "Pumps for High Temperature Liquid Systems," ARS Paper No. 1743-61 (1961)

78. R. W. Atz, "Performance of HNPF Prototype Free-Surface Sodium Pump,'" NAA - SR - 4336 (1960) 
79. R. W. Atz, "Testing of HNPF Freeze-Seal Pumps," NAA-SR-4387 (1960)

80. C. Pfleiderer and H. Petermann, Strömungsmaschinen (Julius Springer, Berlin, and New York, 1964)

81. A. Betz, Introduction to the Theory of Flow Machines (Pergamon Press, New York, 1966)

82. N. Rott and W. S. Lewellen, "Boundary Layers and Their Interactions in Rotating Flows," Progress in Aeronautical Science, Vol 7 (D. Kucheman, ed., Pergamon Press, Oxford, 1966)

83. A. G. Grindell, "Correlation of Cavitation Inception Data for a Centrifugal Pump Operating in Water and in Sodium-Potassium Alloy," J. Basic Eng. p $821-828$ (1960)

84. W. A. Spraker, "The Effects of Fluid Properties on Cavitation in Centrifugal Pumps," Trans. ASME, J. Eng. for Power 87, p 309-318 (1965)

85. A. J. Stepanoff, "Cavitation in Centrifugal Pumps with Liquids Other Than Water," Trans. ASME, J. Eng. for Power 83, p 79-90 (1961)

86. T. Baumeister, "Turbomachinery" in Handbook of Fluid Mechanics (V. L. Streeter, ed., McGraw-Hill Book Co., New York, 1961)

87. A. J. Stepanoff, Centrifugal and Axial Flow Pumps, 2nd ed (John Wiley and Sons, New York, 1957)

88. A. J. Acosta, "Hydrodynamics of Turbomachines," California Institute of Technology Report 79.9 (1965)

89. A. G. Grindell, W. F. Boudreau, and H. W. Savage, "Development of Centrifugal Pumps for Operation with Liquid Metals and Molten Salts at $1100-1500^{\circ} \mathrm{F}, " \mathrm{Nucl}$. Sci. and Eng. 7, p 83-91 (1960)

90. A. J. Stepanoff and H. A. Stahl, "Dissimilarity Laws in Centrifugal Pumps and Blowers," J. Eng. for Power (Trans. ASME) 83 p 381-391 (1961)

91. L. A. Difford, 'Investigation of a High Pressure Liquid Metal Centrifugal Pump," TRW -ER-6632-1-2 (1966)

92. A. I. Leipunskii et al., "Sodium Technology and Equipment of the BN-350 Installation," London Conference on Fast Breeder Reactors, P BNES 5 B/1 (1966)

93. G. Seed and R. J. Brown, "Design Manual for 6,000 gpm Mechanical Sodium Pump," TRG Rep. 1121 (R) (1964) 
94. W. H. Roberts and P. McEwan, "A Sodium-Lubricated Hydrostatic Jet Bearing," TRG Report 1187 (R) (1964).

95. J. Baumier, "Mechanical Pumps for 1 and $10 \mathrm{Mw}$ Test Loops," DRP/ML/FAR - R - 100 (1962)

96. O. Pinkus and B. Sternlicht, Theory of Hydrodynamic Lubrication (McGraw-Hill Book Co., New York, 1961)

97. E. E. Bisson and W. J. Anderson, "Advanced Bearing Technology," NASA -SP - 38 (1964)

98. C. H. T. Pan and J. H. Vohr, "Superlaminar Flow in Bearings and Seals," Proceedings on Superlaminar Flow Bearings on Seals for Process Fluid Lubricated Turbomachinery, NYO-3363-6 (1966)

99. J. Kaye and E. C. Elgar, "Modes of Adiabatic and Diabatic Fluid Flow in an Annulus with an Inner Rotating Cylinder," Trans. ASME 80, p 753-765 (1958)

100. W. Tillman, "Zur Turbulenzentstehung der Strömung zwischen rotierenden Zylindern,"Z. angew. Phys. 13, p 468-475 (1961)

101. E. S. Kulinski and S. Ostrach, "Journal Bearing Velocity Profiles for Small Eccentricity and Moderate Modified Reynolds Numbers," (Trans. ASME) J. Appl. Mech. 34 p 16-22 (1967)

102. H. G. Elrod, C. W. Ng, and C. H. T. Pan, "A Theory for Turbulent Fluid Films and its Application to Bearings," NYO-3363-2 (1965)

103. C. W. Ng and C. H. T. Pan, "A Linearized Turbulent Lubrication Theory," (Trans. ASME) J. Basic Eng. 87, p 675-688 (1965)

104. L. N. Tao, "A Theory of Lubrication in Short Journal Bearings with Turbulent Flow," Trans. ASME 80, p 1734-1740 (1958)

105. D. Muster and B. Sternlicht, eds., Proceedings of the International Symposium on Lubrication and Wear (McCutchan Publishing Corp., Berkeley, 1965)

106. R. R. Donaldson, "Incompressible Journal Bearings with Combined Hydrostatic-Hydrodynamic Action," (Ph. D. Thesis, Department of Mechanical Engineering, Massachusetts Institute of Technology, 1965)

107. J. N. Shinkle and K. G. Hornung, "Frictional Characteristics of Liquid Hydrostatic Journal Bearings," Trans. ASME 87, J. Basic Eng. p $163-169$ (1965) 
108. F. K. Orcutt and E. B. Arwas, "Analysis of Turbulent Lubrication," NASA - CR - 54034 (1964)

109. E. S. Kulinski and S. Ostrach, "A Critical Evaluation of the Status and Trends in High Speed Fluid Film Lubrication," (to be published as a NASA-CR report)

110. J. W. Lund, "Rotor-Bearing Dynamics Design Technology Pt. III," Design Handbook for Fluid Film Type Bearings, AF APL-TR-65-45 Pt. III (1965)

111. H. Poritsky, "Rotor Bearing Dynamics Design Technology Pt. II," Rotor Stability Theory, AFAPL-TR-65-45 Pt. II (1965)

112. N. P. Chironis, "How to Design Bearings for Hydrostatic Lubrication," Product Eng. p 80-86 (July 22, 1963)

112a. R. A. Burton, ed., Bearing and Seal Design in Nuclear Power Machinery, ASME, New York, 1967

113. W. E. Young, "Investigation of Hydrostatic Bearings for Use in High Pressure Cryogenic Pumps," PWA-FR-2145 (1966) and AF-RPL-TR $67-130(1967)$

114. F. K. Orcutt and C. W. Ng, "Steady State and Dynamic Properties of Journal Bearings in Laminar and Superlaminar Flow R egime $s^{\prime \prime}$ (I. Tilting-Pad Bearings, NASA CR-732, 1967) (II. Full Floating Ring Bearings, NASA CR -733 , 1967)

115. E. J. Gunter, "Dynamic Stability of Rotor Bearing Systems," NASA SP $113(1966)$

116. J. Hall and R. Spies, "Research in the Field of Liquid Metal Lubricated Bearings," RDT-TDR-63-4289 Pt I, II, III (1965)

117. P. H. McDonald, "Lubrication Behavior of Liquid Metals," WADC-TR $59-764(1960)$

118. J. Hall et al., "Determination of Working Fluid Lubrication Capability in Journal Bearings," ASD TDR 62-640 Pt I, II (1963)

119. M. Wallace, "Summary Report of Potential Liquid Metal Bearing Materials for SNAP-50/SPUR Pumps," PWAC-468 (1965)

120. D. V. Manfredi, "Summary of SNAP-50/SPUR Pump Bearing Development Program," CN LM-6342 (1965)

121. W. D. Richards, "Hydrodynamic Journal Bearing Tests in Lithium," TIM-915 (1965) 
122. R. A. Burton et al., "Fundamental Investigation of Liquid Metal Lubricated Journal Bearings," SwRI-1228-60 (1965); SwRI-1228-8-16 (1965);

SwRI - 1228-8-20 (1966); SwRI-1 228-p-8-32 (1967); SwRI - $1228-p-8-33$ (1967)

123. R. A. Burton, "Simulation of Turbulent Lubricant Flows in a Large Scale Apparatus," Proceedings on "Superlaminar Flow Bearings and Seals for Process Fluid Lubricated Turbomachinery," NYO 3363-6 (1966)

124. P. H. Stahlhuth and R. J. Trippett, "Liquid Metal Bearing Performance in Laminar and Turbulent Regimes," ASLE Trans. $\underline{5}, p$ 427-436 (1962)

125. P. H. Stahlhuth, "High Temperature Inorganic Lubricant Study," ASDT DR -62-407 (1962)

126. B. Weinberg, "Single Film Journal Bearing Tests in Water," TIM-916 (1965)

127. D. Groll, "Summary of Fluid Film Bearing Analysis," TIM-917 (1965)

128. C. Merz, "Straddle Bearing Rotor Dynamics Tests in Water," TIM-836 (1964)

129. D. A. Newey, "Overhung Rotor Dynamics Tests Using Hydrostatic Water Bearings," TIM-874 (1965)

130. L. Knudsen, "Single Hybrid Journal Bearing Tests in Water," TIM-925 (1965)

131. L. Knudsen, "Thrust Bearing Tests in Water," TIM-926 (1965)

132. W. Richards, "Ball Bearing Test in Lithium," TIM-923 (1965)

133. R. Beichel et al., "Hydrostatic Bearing Feasibility Program," AFRPLTR $-65-120$ (1965)

134. D. R. Grieser, "Liquid Metal Lubricated Bearing Instrumentation," Pt. II, AF APL-TR 65-31 (1967)

135. H. E. Nichols et al., "Hydrodynamic Journal Bearing Program," NASA CR 72175 (1966)

136. F. T. Schuller et al., "Experiments with Hydrodynamic Journal Bearings of Various Materials and Design," Proceedings on Superlaminar Flow Bearings on Seals for Process Fluid Lubricated Turbomachinery, NYO-3363-6(1966)

137. F. T. Schuller and W. J. Anderson, "Operation of Hydrodynamic Journal Bearings in Sodium and Temperatures to $800^{\circ} \mathrm{F}$ and Speed s to $12,000 \mathrm{rpm}$," NASA - TN-D-3928 (1967) 
138. W. D. Waldron and E. B. Arwas, "Preliminary Study of the Liquid Metal Loop and Test Rig for Phase II of the Investigation of Liquid Metal Lubricated Bearings and Rotor Bearings Dynamics," MTI 64TR72 (Rev. 2) (1965)

139. J. Vohr, "Experimental Study of Superlaminar Flow Between NonConcentric Rotating Cylinders," NASA-CR-749 (1967)

140. A. E. King, "Developing Screw Type Shaft Seals for Potassium Environment," Research and Development 17, p 48-57 (1966)

141. A. I. Golubier, 'Studies on Seals for Rotating Shafts of High Pressure Pumps," Wear $\underline{8}$, p $270-288$ (1965)

142. L. P. Ludwig et al., "Gas Ingestion and Sealing Capacity of Helical Groove Fluid Film Seal (Viscoseal) Using Sodium and Water as Sealed Fluids," NASA - TND-3348 (1966)

143. J. Zuk et al., "Experimental and Theoretical Study of the Viscoseal," Proceedings of Technical Meeting on Superlaminar Flow Bearings and Seals for Process Fluid Lubricated Turbomachinery, NYO.3363-6 (1966)

144. W. K. Stair, "The Effect of Turbulence on Viscoseal Performance," Proceedings of Technical Meeting on Superlaminar Flow Bearings and Seals for Process Fluid Lubricated Turbomachinery, NYO 3363-6 (1966)

145. W. K. Stair, "Analysis of the Visco Seal," NASA-CR -285 (1965)

146. J. C. Hudelson, "Noncontact Pump-Seals for the Space Vacuum," SpaceAeron. 48, No.5, p $110-112$ )1967)

147. G. M. Wood et al., "Centrifugal Dynamic Shaft Seals," Mech. Eng. 86, p 48-55 (November 1964) also ASME paper 63-W A-167 (1964)

148. E. F. Boon and S. E. Tal, "Hydrodynamische Dichtung fur Rotierende Wellen," Chem. Ing. Techn. 31, p 202-212 (1959)

149. W. K. Stair, "The Effect of Turbulence on Visco-Seal Performance," Proceedings on Superlaminar Flow Bearings on Seals for Process Fluid Lubricated Turbomachinery, NYO-3363-6 (1966)

150. J. M. McGrew and J. D. McHugh, "Analysis and Test of the Screw Seal in Laminar and Turbulent Operation," Trans. ASME J. Basic Eng. 87, p 153-162 (1965)

151. A. F. Emery and D. A. Bailey, "Heat Transfer to Fully Developed Liquid Metal Flow in Tubes," Trans. ASME, J. Heat Transf. 89, p 272-273 (1967) 
152. R. K. McMordie and A. F. Emery, "A Numerical Solution for Laminar Flow Heat Transfer in Circular Tubes With Axial Conduction and Developing Thermal and Velocity Fields," Trans. ASME, J. Heat Transf. 89, p $11-16$ (1967)

153. R. P. Stein, "Liquid Metal Heat Transfer," Advances in Heat Transfer, Vol. III (J.P. Hartnett, ed., Academic Press, New York, 1966)

154. W. M. Kays, Convective Heat and Mass Transfer, (McGraw-Hill Book Co., New York, 1966)

155. O. E. Dwyer, "Eddy Transport in Liquid Metal Heat Transfer," AIChE J. $\underline{9}$, p $261-268$ (1963)

156. R. G. Palmer and A. Platt, Fast Reactor, (Temple Press, London, 1961)

157. R. N. Lion, "Heat Transfer to Liquid Metals," TID-278 (1949)

158. B. Lubarsky and S. J. Kaufman, "Review of Experimental Investigation of Liquid Metal Heat Transfer," NACA Report 1270 (1956)

159. O. E. Dwyer, "Recent Developments in Liquid Metal Heat Transfer," Atomic Energy Review 4, p $3-92$ (1966)

160. V. I. Subbotin et al., "Study of Heat Transfer to Liquid Sodium in Tubes," Atomn. Energ. 13, p 380-382 (1962)

161. N. I. Buleev, "Theoretical Model of the Mechanism of Turbulent Exchange in Fluid Flows," AERE Trans. 957 (1962)

162. V. I. Subbotin et al., 'Heat Exchange in Fuel Elements of Liquid Metal Cooled Reactors," Third Conference on Peaceful Uses of Atomic Energy, 1964, P 328 (Translation: NASA-TT-F 10776)

163. V. I. Subbotin et al., "Experimental Study on Models of the Temperature Fields of Fuel Elements," JPRS 31245 (1965)

164. R. Herrick, "Liquid Metal Heat Transfer by Forced Convection," (A Literature Survey), TRG Rep. 546 (R) (1964)

165. R. M. Inman, "Theoretical Investigation of Turbulent Liquid Metal Heat Transfer in Channels," NASA TNK 3692 (1966)

166. R. M. Inman, 'Heat Transfer to Liquid Metal Flow in a Round Tube or Flat Duct with Heat Sources in the Fluid Stream," NASA-TN-D-3473 (1966)

167. L. S. Kokorev, "Relation Between the Coefficients of Turbulent Heat and Momentum Exchange in Turbulent Flow of Liquid Metal, "AEC-tr-6554 (1963) 
168. T. V. Zablotskaya, "Heat Transfer for Turbulent Flow in Pipes for Liquids with Small Prandtl Numbers (Pr $\ll$ 1)," AEC-tr-6554 (1963)

169. A. I. Leont'ev, "Heat and Mass Transfer in Turbulent Boundary Layers," Advances in Heat Transfer, Vol. III (J. P. Hartnett, ed., Academic Press, New York, 1966)

170. J. O. Hinze, Turbulence (McGraw-Hill Book Co., New York, 1959)

171. J. L. Lumley, "The Applicability of Turbulence Research to the Solution of Internal Flow Problems," Fluid Dynamics of Internal Flow, (G. Sovran, ed., Elsevier Publishing Co., Amsterdam, London, New York, 1967) p $152-169$

172. J. L. Lumley, "Turbulence in Liquids," Encyc. Dict. of Phys. (Pergamon Press, Oxford, 1963)

173. J. C. Rotta, "Turbulent Boundary Layers in Incompressible Flow," Progress in Aero-Sciences, Vol. II (Pergamon Press, New York, 1962)

174. G. Kleinstein, "Generalized Law of the Wall and Eddy Viscosity Model for Well Boundary Layers," AIAA Journ. 5, p 1402-1407 (1967)

175. H. Kato et al., "Turbulent Heat Transfer in Smooth Pipes at High Prandtl Numbers," Proceedings of Eleventh Congress of Applied Mechanics, Munich, 1964 (Julius Springer, Berlin, Heidelberg, New York, 1966)

176. M. F. Taylor, "Correlation of Friction Coefficients for Laminar and Turbulent Flow with Ratios of Surface to Bulk Temperature from 0.35 to 7.35," NASA TR-R-267 (1967)

177. P. Miller et al., 'Heat Transfer to Water Flowing Parallel to a Rod Bundle," Nuclear Engineering and Science Congress, Vol I (Pergamon Press, New York, 1957) p 350-365

178. S. S. Kutateladze et al., Liquid Metal Heat Transfer Media (Consultants Bureau, New York, 1959)

179. H. E. Brown et al., "Temperature and Velocity Distribution and Transfer of Heat in a Liquid Metal," Trans. ASME 79, p 279-285 (1957)

180. R. G. Deissler and M. F. Taylor, "Analysis of Axial Turbulent Flow and Heat Transfer through Banks of Rods and Tubes," Reactor Heat Transfer Conference of 1956, TID-7529, Pt. 1, Book 2, p 416-461 (1956)

181. V. M. Borshanskii et al., "Heat Transfer and Hydraulic Resistance in the Motion of Liquid Metals in Circular Tubes," AEC-tr-6554 (1963) 
182. E. R. G. Eckert et al., "Heat Transfer, A Review of Current Literature," Int. J. Heat Transfer $\underline{3}$, p 222-248 (1961)

183. S. Ostrach, "An Analysis of Laminar Free Convection Flow and Heat Transfer About a Flat Plate Parallel to the Direction of the Generating Body Force," NACA-TR-1111 (1953)

184. S. Ostrach, "Laminar Flow with Body Forces," Theory of Laminar Flows (F. K. Moore, ed.), High Speed Aerodynamics and Jet Propulsion, Vol. IV, (Princeton University Press, 1964)

185. A. J. Ede, "Advances in Free Convection," Advances in Heat Transfer, Vol. IV (J. P. Hartnett, ed.) (Academic Press, New York, 1967)

186. L. S. Tong, Boiling Heat Transfer and Two-Phase Flow (John Wiley and Sons, New York, 1965)

187. A. J. Stepanoff, Pumps and Blowers, Two-Phase Flow, (John Wiley and Sons, New York, 1965)

188. W. M. Rohsenow and J. A. Clark, 'Heat Transfer and Pressure Drop Data for High Heat Flux Densities to Water at High Sub-critical Pressures," Heat Transfer and Fluid Mechanics Institute, 1951, (Stanford Univ. Press 1951) p $193-207$

189. H. K. Forster and N. Zuber, "Dynamics of Vapor Bubbles and Boiling Heat Transfer," AIChE J. 1, p $531-535$ (1955)

190. S. G. Camack and H. K. Forster, "Test of a Heat Transfer Correlation for Boiling Liquid Metals," Jet Prop. 27, p 1104 (1957)

191. N. Madson and C. F. Bonilla, 'Heat Transfer to Sodium-Potassium Alloy in Pool Boiling," Chem. Eng. Progr. Symp., Ser. 56, p 251-259 (1960)

192. R. C. Martinelli and D. B. Nelson, "Prediction of Pressure Drops During Forced Circulation Boiling of Water," Trans. ASME $\underline{70}$, p $695-702$ (1948)

193. C. J. Baroczy, "A Systematic Correlation for Two Phase Pressure Drop," Chem. Eng. Progr. Symp., Ser. 62, p 232-247 (1966)

194. C.J. Baroczy, "Correlation of Liquid Fraction - Two Phase Flow with Application to Liquid Metals," NAA-SR-8171 (1963)

195. W. H. Lowdermilk et al., "Investigation of Boiling Burnout and Flow Stability for Water Flowing in Tubes, "NACA-TN 4382 (1958) 
196. K. Hargraeves et al., "Cavitation Suppression in a Hydraulic Resistance for the Sodium Pump Rig," TRG-Memo-3104 (R) (1966)

197. R. Kobayashi, "Effect of Cavitation on the Discharge Coefficient of Standard Flow Nozzles," ASME J. Basic Eng. 89, p 677-685 (1967)

198. M. J. Robinson, "Cavitation Damage Characteristics in Water and Mercury From Studies in a Cavitating Venturi," NASA-CR-76027 (1966)

199. G. G. Hirs, "Turbulent Lubricant Film," SR-TN 6701-08 (1967)

200. R. Garcia and F. G. Hammit, "Cavitation Damage and Correlations with Material and Fluid Properties," Trans. ASME J. Basic Eng. $\underline{89}$, $753-763$ (1967)

201. A. W. Marris, "Theory of the Bathtub Vortex," ASME J. Appl. Mech. $\underline{89}$, p $11-15$ (1967)

202. M. Y. M. Kamel, "The Effect of Swirl on the Flow of Liquids Through Bottom Outlets," ASME P 64-W A/FE-37 (1964)

203. F. T. Dodge, "Liquid Rotation and Vortexing During Draining," The Dynamic Behavior of Liquids in Moving Containers (H. N. Abramson, ed.), NASA -SP 106 (1966)

204. H. M. Abramson, "Some Studies of Liquid Roation and Vortexing in Rocket Propellent Tanks," NASA TN-D 1212 (1962)

205. M. Harada, "On the Generalized Bath-Tub Vortex," ASME J. Basic Eng. 89, p 617-623 (1967)

206. M. G. Hall, "The Structure of Concentrated Vortex Cores," Progress in Aeronautical Science, Vol. 7 (D. Küchemann, ed., Pergamon Press, Oxford, 1966)

207. R. Wille, "On Unsteady Flows and Transient Motions," Progress in Aeronautical Science, Vol. 7 (D. Kuchemann, ed., Pergamon Press, Oxford, 1966)

208. L. K. Spink, Principles and Practice of Flow Meter Engineering, 9th Ed. (The Foxboro Co., Foxboro, 1967)

209. W. J. Clark, Flow Measurement (Pergamon Press, New York, 1965)

210. R. C. Dean, Jr., "Separation and Stall," Handbook of Fluid Dynamics (V. L. Streeter, ed., McGraw-Hill Book Co., New York, 1961) 
211. R. Garcia et al., "Acoustic Noise From a Cavitating Venturi," ORA-06110-1-T (1964)

212. F. Numadis et al., "Effect of Cavitation on the Accuracy of HerschelType Venturi Tubes," ASME J. Basic Eng. 84, p 351-362 (1962)

213. J. Parmalian, Water Hammer Analysis (Dover Press, New York, 1955)

214. G. R. Rich, Hydraulic Transients (Dover Press, New York, 1962)

215. V. L. Streeter (ed.), International Symposium on Water Hammer in Pumped Storage Projects, ASME, New York (1965)

216. L. L. Kalishevskii and S. V. Selikhovkin, "Some Results of Investigating Unsteady Turbulent Flow," Teploenergetica 14, p 69-72 (1967)

217. H. M. Paynter, Fluid Transients in Engineering Systems, Handbook of Fluid Dynamics (V. L. Streeter, ed., McGraw-Hill Book Co., New York, 1961)

218. I. P. Ginzburg, Applied Fluid Dynamics, NASA TTF 4 (1963)

219. D. Burgreen et al., "Vibration of Rods Induced by Water in Parallel Flow," Trans. ASME 80, p 991-1001 (1958)

220. Y. N. Chen, "Flow Induced Vibration and Noise in the Bank Heat Exchangers Due to Von Karman Streets, " ASME Vibration Conference, P 67, NBR 48 (1967)

221. J. S. McDonald, "Analysis of Turbulence Induced Vibrations of Tubular Immersion Heaters," NAA-SR-Memo-6576 (1961)

222. G. H. Toebes, "Flow Induced Structural Vibrations," ASCE of J. Eng. Mech., pp 39-66 (December 1965)

223. M. P. Paidoussis, "The Amplitude of Fluid-Induced Vibrations of Cylinders in Axial Flow," AECL 2225 (1965)

224. E. P. Quinn, "Vibration of SEFOR Fuel Rods in Parallel Flow," GEAP 4966 (1965)

225. E. P. Quinn, "Vibration of Fuel Rods in Parallel Flow," GEAP-4059 (1962)

\section{LMEC -68-4}

